\title{
Hermitian Clifford analysis on superspace
}

\author{
Hennie De Schepper, Alí Guzmán Adán, Frank Sommen \\ Clifford Research Group, Department of Mathematical Analysis, Faculty of Engineering and \\ Architecture, Ghent University, Galglaan 2, 9000 Ghent, Belgium.
}

\begin{abstract}
In this paper we first recall the proper algebraic framework, i.e. the radial algebra, needed to extend Hermitian Clifford analysis to the superspace setting. The fundamental objects for this extension then are introduced by means of an abstract complex structure on the Hermitian radial algebra. This leads to a natural representation of this Hermitian radial algebra on superspace.
\end{abstract}

Keywords. Hemitian Clifford analysis, Superspace, Radial algebra Mathematics Subject Classification (2010).

\section{Introduction}

Clifford analysis nowadays is a well established mathematical discipline constituting a natural refinement of harmonic analysis. In its most simple setting, it focusses on the null solutions of the Dirac operator $\partial_{\underline{x}}=\sum_{j=1}^{m} e_{j} \partial_{x_{j}}$, where the elements $\left(e_{1}, \ldots, e_{m}\right)$ form an orthonormal basis for the Euclidean space $\mathbb{R}^{m}$ leading to the construction of the real Clifford algebra $\mathbb{R}_{0, m}$. This setting is known as the orthogonal case since the fundamental group leaving the Dirac operator invariant is $\operatorname{Spin}(m)$ which is a double covering of $\operatorname{SO}(m)$.

By taking the dimension even, say $2 m$, and introducing a so-called complex structure $J \in \mathrm{SO}(2 m)$, the fundamental elements of Hermitian Clifford analysis arise in a natural way from the orthogonal setting. The Hermitian case focusses on $h$-monogenic functions, $h$-monogenicity being expressed by means of two mutually adjoint Dirac operators which are invariant under the realization of the unitary group $\mathrm{U}(m)$ in $\operatorname{Spin}(2 m)$. Indeed, the action of the projection operators $\frac{1}{2}(1 \pm i J)$ on the initial orthonormal basis $\left(e_{1}, \ldots, e_{2 m}\right)$ leads to the Witt basis elements $\left(\mathfrak{f}_{j}: j=1, \ldots, m\right)$ and $\left(\mathfrak{f}_{j}^{\dagger}: j=1, \ldots, m\right)$, producing a direct sum decomposition of $\mathbb{C}^{2 m}$ in two components. The elements of $\mathrm{SO}(2 m)$ leaving those subspaces invariant generate a subgroup which is doubly covered by a subgroup of $\operatorname{Spin}(2 m)$ denoted $\operatorname{Spin}_{J}(2 m)$, and being isomorphic with the unitary group. The Hermitian Dirac operators $\partial_{\underline{z}}$ and $\partial_{\underline{z}}^{\dagger}$ are given by projection of the gradient on the aforementioned invariant subspaces, whence they are invariant under the action of $\operatorname{Spin}_{J}(2 m)$.

This Hermitian decomposition has been thoroughly studied in several papers, see for example [2, 3]. Results concerning spherical monogenics, invariant differential operators, a Fischer decomposition and integral representation formulae (Bochner-Martinelli, Cauchy) have already been obtained, see [1, 2, 3, 7]. Furthermore, Hermitian Clifford analysis was addressed in [13] where several complex operators $\partial_{\underline{z}}, \partial_{\underline{z}}^{\dagger}, \partial_{\underline{u}}, \partial_{\underline{u}}^{\dagger}, \ldots$ were considered giving rise to new syzygy complexes. Those results motivated for the first time the use of the radial algebra in the Hermitian setting which is independent of the choice of the dimension parameter.

The radial algebra framework is defined through an algebra $R(S)$ generated by a set $S$ of abstract vector variables $x, y, \ldots$, where classical notions of Clifford analysis are reintroduced axiomatically. For example, Dirac operators are axiomatically defined as endomorphisms on $R(S)$, more precisely as a vector derivative denoted by $\partial_{x}, x \in S$. A first account on such an axiomatisation can be found in [15] and was inspired by the work on "geometric calculus" presented by Hestenes and Sobczyk [12].

The radial algebra possesses some important properties, of which the most powerful most probably is its independence of any particular dimension $m$, which is now abstractly defined as a complex parameter 
stemming from the evaluation $\partial_{x}[x]=m$. In addition, $R(S)$ is independent of the choice of an underlying vector space $V$ to which the vector variables belong and of any chosen quadratic form on $V$. Hence the polynomial Clifford representation turns out to be only one of the possible frameworks where the radial algebra axioms are fulfilled, see for example [8, 15], giving rise to important applications of the radial algebra setting in the study of the Fischer decomposition and the Dirac complex $([4,13,14])$.

In this paper, we are interested in using the radial algebra for the introduction of a theory of Hermitian Dirac operators in superspace. This construction is inspired by the succesful extension of orthogonal Clifford analysis to superspace, see $[5,6,16,17,18,19]$. In particular the radial algebra was proven to be an efficient tool for giving a meaning to vector spaces of negative dimension, and defining the fundamental objects of Clifford analysis, such as vector variables and vector derivatives, in such a case.

In the paper [8] we have introduced the so-called Hermitian radial algebra in order to fix the rules that allow a canonical extension of Hermitian Clifford analysis to superspace. The objective of the current paper now is to explicitly introduce the fundamental objects of Hermitian Clifford analysis in the superspace setting. In particular, we will establish the notion of a complex structure, as well as its realization as a bivector, which then will lead to the Witt basis, the Hermitian vector variables, the Hermitian vector derivatives and the complex Euler operators in superspace. These notions constitute the starting point for the study of this representation of the Hermitian radial algebra. In future work we plan to address some other classical issues such as the underlying group structure, spin representations, invariance of the Dirac operators under spin actions and also Bochner-Martinelli formulae in the Hermitian superspace setting.

The outline of the paper is as follows. We first present a brief overview of the radial algebra setting for the Euclidean and the Hermitian cases, respectively. Next, we establish the corresponding representation of the radial algebra in superspace by means of the definitions of the corresponding radial algebra endomorphisms in this setting. In particular, the vector multipliers give rise to a natural way of introducing a complex structure on superspace which is thoroughly studied in Section 5. Moreover, it is proven there that all defined objects satisfy the abstract relations provided by the Hermitian radial algebra. Finally, in Section 6 we give explicit expressions for the complex Hermitian vector variables, Dirac operators and directional derivatives. Some final remarks are given in Section 7 about the future direction of this research: in the two forthcoming papers $[9,10]$, we will properly introduce the Spin group in superspace in order to give a meaning to its subgroup $\operatorname{Spin}_{J}$, consisting of elements which commute with the complex structure.

\section{Preliminaries on radial algebras}

Given a set $S$ of symbols $x, y, \ldots$ we define the radial algebra $R(S)$ as the associative algebra over $\mathbb{R}$ freely generated by $S$ and subject to the defining axiom

$$
[\{x, y\}, z]=0 \quad \text { for any } x, y, z \in S
$$

where $\{a, b\}=a b+b a$ and $[a, b]=a b-b a$. Elements in $S$ are called abstract vector variables.

Axiom (A1) means that the anti-commutator of two abstract vector variables is a scalar, i.e. a quantity that commutes with every other element in the algebra. It is clearly inspired by the similar property for Clifford vector variables.

Definition 1. A radial algebra representation is an algebra homomorphism $\Psi: R(S) \rightarrow \mathfrak{A}$ from $R(S)$ into an algebra $\mathfrak{A}$. The term representation also refers to the range $\Psi(R(S)) \subset \mathfrak{A}$ of that mapping.

The easiest and most important example of radial algebra representation is the algebra generated by standard Clifford vector variables. The classical Clifford polynomial representation is defined for $S=\left\{x_{1}, \ldots, x_{s}\right\}$ by considering the mapping

$$
x_{j} \rightarrow \underline{x}_{j}=\sum_{\ell=1}^{m} x_{j, \ell} e_{\ell} \quad\left(e_{j} e_{k}+e_{k} e_{j}=-2 \delta_{j, k}\right),
$$

where the elements $x_{j, \ell}(\ell=1, \ldots, m)$ are real variables generating a polynomial algebra $\mathbb{R}\left[x_{1,1}, \ldots, x_{s, m}\right]$ and the elements $e_{\ell}(\ell=1 \ldots, m)$ generate the Clifford algebra $\mathbb{R}_{0, m}$. The correspondences $(1)$ naturally 
extend to an algebra homomorphism from $R(S)$ to the algebra $\mathbb{R}\left[x_{1,1}, \ldots, x_{s, m}\right] \otimes \mathbb{R}_{0, m}$ of Cliffordvalued polynomials. Indeed, for any two vector variables $\underline{x}_{j}, \underline{x}_{k}$ we have that $\left\{\underline{x}_{j}, \underline{x}_{k}\right\}=\underline{x}_{j} \underline{x}_{k}+\underline{x}_{k} \underline{x}_{j}=$ $-2 \sum_{\ell=1}^{m} x_{j, \ell} x_{k, \ell}$ is a central element in $\mathbb{R}\left[x_{1,1}, \ldots, x_{s, m}\right] \otimes \mathbb{R}_{0, m}$. In this case, elements in $S$ are represented by vector variables $\underline{x}_{j}$ defined in the underlying vector space $V=\mathbb{R}^{m}$. The set $\underline{S}:=\left\{\underline{x}_{j}: j=1 \ldots, s\right\}$ of Clifford vector variables established by (1) generates the Clifford polynomial representation $R(\underline{S}) \subset$ $\mathbb{R}\left[x_{1,1}, \ldots, x_{s, m}\right] \otimes \mathbb{R}_{0, m}$. A thorough study of this representation can be found in [15].

The simplest case is obtained for $S=\{x\}$ in which case $R(S)$ is mapped into the real algebra of polynomials of the form $\underline{x}^{s}$, where $\underline{x}^{2 s}=\left(-|\underline{x}|^{2}\right)^{s}$ and $\underline{x}^{2 s+1}=\underline{x}\left(-|\underline{x}|^{2}\right)^{s}$. To have a non-trivial radial algebra for Clifford analysis, the above set of radially symmetric functions $\underline{x}^{s}$ is too limited. One needs at least something of the form $S=\{x, u\}$ where the corresponding Clifford vectors are given by $x \rightarrow \underline{x}=\sum_{k=1}^{m} x_{k} e_{k}$ and $u \rightarrow \underline{u}=\sum_{k=1}^{m} u_{k} e_{k}$. Here the vector $x$ is considered as the variable vector and $u$ as a parameter vector. The elements of the algebra $R(S)$ clearly have the form $F=A+B x+C u+D x \wedge u$ where $A, B, C, D$ are polynomials of the three variables $x^{2}, u^{2}, x \cdot u$ and $x \wedge u=\frac{1}{2}[x, u]$. With these two vector variables one can abstractly produce the so-called zonal monogenic polynomials, see [11]. More in general a typical choice for $S$ would be $S=\left\{x_{1}, \ldots, x_{s}\right\} \cup\left\{u_{1}, \ldots, u_{t}\right\}$, where the variables $x_{j}$ are the vector variables on which functions depend and $u_{j}$ are extra parameter vectors. This choice for $S$ is used when studying monogenic functions in several vector variables $x_{1}, \ldots, x_{s}$, see also [4].

The main difference between Clifford algebra and radial algebra lies in the fact that the abstract vector variables $x \in S$ have a merely symbolic nature; they are not vectors belonging to an a priory defined vector space $V$ with some dimension $m$ and some quadratic form on it. The radial algebra is a generalization of both polynomial algebras and Clifford algebras. Indeed, it suffices to identify a subset $\left\{u_{1} \ldots, u_{m}\right\}$ of parameter vectors with the Clifford generators, i.e. $u_{\ell} \mapsto e_{\ell}, \ell=1, \ldots, m$. In this case, the inner product $-\frac{1}{2}\left\{x_{j}, u_{\ell}\right\}$ is mapped to $-\frac{1}{2}\left\{\underline{x}_{j}, \underline{u}_{\ell}\right\}=x_{j, \ell}(j=1, \ldots, s ; \ell=1, \ldots, m)$, which generates the polynomial algebra $\mathbb{R}\left[x_{1,1}, \ldots, x_{s, m}\right]$.

We can abstractly define the notion of scalar subalgebra (i.e. the algebra of all commuting elements) by

$$
R_{0}(S):=\operatorname{Alg}_{\mathbb{R}}\{\{x, y\}: x, y \in S\}
$$

and the wedge product of vectors (for example $x_{1}, \ldots, x_{k} \in S$ ) by

$$
x_{1} \wedge \ldots \wedge x_{k}=\frac{1}{k !} \sum_{\pi} \operatorname{sgn}(\pi) x_{\pi(1)} \cdots x_{\pi(k)} .
$$

Then abstract $k$-vectors are defined as linear combinations of elements of the form $f x_{1} \wedge \ldots \wedge x_{k}$ where $f \in R_{0}(S)$ and $x_{1}, \ldots, x_{k} \in S$. Denoting by $R_{k}(S)$ the space of all $k$-vectors, it can be proven as a consequence of (A1) that (see [15])

$$
R(S)=R_{0}(S) \oplus R_{1}(S) \oplus R_{2}(S) \oplus \cdots
$$

On this radial algebra we can define several endomorphisms. Here we will only give an overview of those which are relevant in the current setting. For a more detailed account we refer the reader to $[8,15]$.

- The main involution $\tilde{\cdot}$ and the conjugation - are respectively defined as the linear maps satisfying:

$$
\begin{gathered}
\overline{a b}=\bar{b} \bar{a}, \quad \widetilde{a b}=\widetilde{a} \widetilde{b}, \quad a, b \in R(S) \\
\bar{x}=\widetilde{x}=-x, \quad x \in S .
\end{gathered}
$$

- The vector multipliers $x[\cdot]$ and $x \mid[\cdot]$ are defined for every $x \in S$ by

$$
x: F \rightarrow x F, \quad x \mid: F \rightarrow \widetilde{F} x, \quad F \in R(S) .
$$

- The directional derivatives $D_{y, x}$ are defined for every pair $x, y \in S$ by

$$
\begin{array}{ll}
\text { (DD1) } & D_{y, x}[F G]=D_{y, x}[F] G+F D_{y, x}[G], \quad F, G \in R(S), \\
\text { (DD2) } & D_{y, x}[z]=\delta_{x, z} y, \quad z \in S .
\end{array}
$$

The operator $D_{x, x}$ corresponds to the classical Euler operator $E_{x}$ which measures the degree of homogeneity with respect to the vector variable $x \in S$. 
- The left and right actions of the vector derivative $\partial_{x}$ are defined for every $x \in S$ by the axioms

(D1)

$$
\begin{array}{ll}
\text { (D1) } & \partial_{x}[f F]=\partial_{x}[f] F+f \partial_{x}[F], \\
& {[f F] \partial_{x}=F[f] \partial_{x}+f[F] \partial_{x}, \quad f \in R_{0}(S), \quad F \in R(S),} \\
\text { (D2) } \quad & \left\{\begin{array}{l}
\partial_{x}[x G]=\partial_{x}[x] G, \quad[G x] \partial_{x}=G[x] \partial_{x}, \\
\partial_{x}[G]=[G] \partial_{x}=0,
\end{array}\right. \\
\text { (D3) } \quad\left[\partial_{x} F\right] \partial_{y}=\partial_{x}\left[F \partial_{y}\right], \quad x, y \in S, & \text { (D4) } \quad \partial_{x}\left[x^{2}\right]=\left[x^{2}\right] \partial_{x}=2 x, \quad \partial_{x}\{x, y\}=\{x, y\} \partial_{x}=2 y, \quad y \neq x .
\end{array}
$$

From these axioms it follows that $\partial_{x}[x]=[x] \partial_{x}$ is a real constant independent of $x$.

- The operator $B:=\left\{\partial_{x}, x \mid\right\}$ is independent of $x \in S$; it can be also written as $B:=-\left\{\partial_{x} \mid, x\right\}$ where:

$$
\partial_{x} \mid: F \rightarrow[\widetilde{F}] \partial_{x} .
$$

In [8] the notion of a complex structure was introduced on the radial algebra framework; we recall its definition and main properties. Consider a bijective map $J: \mathcal{S} \rightarrow J(\mathcal{S})$, producing a disjoint copy of the set of abstract vector variables $\mathcal{S}$, i.e. $\mathcal{S} \cap J(\mathcal{S})=\emptyset$. Now consider the algebra $R(\mathcal{S} \cup J(\mathcal{S}), \mathbf{B})$ generated over the real numbers by $\mathcal{S}, J(\mathcal{S})$ and a fixed element $\mathbf{B}$. We say that $J$ is a complex structure over $\mathcal{S}$ if the following axioms are fulfilled on $R(\mathcal{S} \cup J(\mathcal{S}), \mathbf{B})$.
(AH1)
$\{x, y\}=\{J(x), J(y)\}$
$\{J(x), y\}=-\{x, J(y)\} \quad x, y \in \mathcal{S}$,
(AH2)
$\{x, y\}$ and $\{J(x), y\}$ are central elements for all $x, y \in \mathcal{S}$,
(AH3)

$$
[\mathbf{B}, x]=-2 J(x),
$$
$[\mathbf{B}, J(x)]=2 x, \quad x \in \mathcal{S}$.

As before, we will use the notation $R_{0}(\mathcal{S} \cup J(\mathcal{S})):=\operatorname{Alg}_{\mathbb{R}}\{\{x, y\},\{J(x), y\}: x, y \in \mathcal{S}\}$ for the scalar subalgebra of $R(\mathcal{S} \cup J(\mathcal{S}), \mathbf{B})$.

The map $J$ can be extended from $\mathcal{S}$ to an algebra automorphism on $R(\mathcal{S} \cup J(\mathcal{S}), \mathbf{B})$ by linearity and by the rules

$$
\begin{aligned}
& J(F G)=J(F) J(G), \quad F, G \in R(\mathcal{S} \cup J(\mathcal{S}), \mathbf{B}), \\
& J(\mathbf{B})=\mathbf{B}, \quad J(J(x))=-x, \quad x \in \mathcal{S} .
\end{aligned}
$$

Observe that the axioms (AH1) -(AH3) together with the rules (2)-(3) ensure that $J$ is an isomorphism between the radial algebras $R(\mathcal{S})$ and $R(J(\mathcal{S}))$.

The conjugation can be extended from $R(\mathcal{S})$ to $R(\mathcal{S} \cup J(\mathcal{S})$, B $)$ using the axioms

$$
\overline{\mathbf{B}}=-\mathbf{B}, \quad \bar{x}=-x, \quad \overline{J(x)}=-J(x), \quad x \in \mathcal{S}
$$

and

$$
\overline{a b}=\bar{b} \bar{a}, \quad a, b \in R(\mathcal{S} \cup J(\mathcal{S}), \mathbf{B}) .
$$

In order to define the action of the complex structure $J$ on the vector derivatives, the definitions of the formal operators $\partial_{x}$ and $\partial_{J(x)}$ have to be extended from their respective initial radial algebras $R(\mathcal{S})$ and $R(J(\mathcal{S}))$ to $R(\mathcal{S} \cup J(\mathcal{S}), \mathbf{B})$. This is accomplished through the following axioms:

(DH1)

$$
\begin{aligned}
& \partial_{x}[f F]=\partial_{x}[f] F+f \partial_{x}[F], \quad \partial_{J(x)}[f F]=\partial_{J(x)}[f] F+f \partial_{J(x)}[F], \\
& {[f F] \partial_{x}=F[f] \partial_{x}+f[F] \partial_{x}, \quad[f F] \partial_{J(x)}=F[f] \partial_{J(x)}+f[F] \partial_{J(x)},} \\
& f \in R_{0}(\mathcal{S} \cup J(\mathcal{S})), \quad F \in R(\mathcal{S} \cup J(\mathcal{S}), \mathbf{B}),
\end{aligned}
$$

(DH2)

$$
\begin{aligned}
& \partial_{x}[G]=[G] \partial_{x}=\partial_{J(x)}[G]=[G] \partial_{J(x)}=0, \\
& \left\{\begin{array} { l } 
{ \partial _ { x } [ x G ] = \partial _ { x } [ x ] G , } \\
{ \partial _ { x } [ J ( x ) G ] = \partial _ { x } [ J ( x ) ] G , } \\
{ \partial _ { J ( x ) } [ x G ] = \partial _ { J ( x ) } [ x ] G , } \\
{ \partial _ { J ( x ) } [ J ( x ) G ] = \partial _ { J ( x ) } [ J ( x ) ] G , }
\end{array} \left\{\begin{array}{l}
{[G x] \partial_{x}=G[x] \partial_{x},} \\
{[G J(x)] \partial_{x}=G[J(x)] \partial_{x},} \\
{[G x] \partial_{J(x)}=G[x] \partial_{J(x)},} \\
{[G J(x)] \partial_{J(x)}=G[J(x)] \partial_{J(x)},}
\end{array},\left\{\begin{array}{l}
\partial_{x}[x J(x) G]=\partial_{x}[x J(x)] G, \\
\partial_{J(x)}[x J(x) G]=\partial_{J(x)}[x J(x)] G, \\
{[G x J(x)] \partial_{x}=G[x J(x)] \partial_{x},} \\
{[G x J(x)] \partial_{J(x)}=G[x J(x)] \partial_{J(x)},}
\end{array}\right.\right.\right. \\
& \text { for } G \in R((\mathcal{S} \backslash\{x\}) \cup J(\mathcal{S} \backslash\{x\}), \mathbf{B}),
\end{aligned}
$$


(DH3) $\partial_{x}[x]=[x] \partial_{x}=\mathfrak{m}=\partial_{J(x)}[J(x)]=[J(x)] \partial_{J(x)}$, where $\mathfrak{m}$ is the abstract dimension of $R(\mathcal{S})$,

$\partial_{x}[J(x)]=-[J(x)] \partial_{x}=2 \mathbf{B}=-\partial_{J(x)}[x]=[x] \partial_{J(x)}$.

(DH4)

$$
\begin{aligned}
& \begin{cases}\partial_{x}\left[x^{2}\right]=\left[x^{2}\right] \partial_{x}=2 x, & \partial_{J(x)}\left[x^{2}\right]=\left[x^{2}\right] \partial_{J(x)}=2 J(x), \\
\partial_{x}[x J(x)]=(\mathfrak{m}+2) J(x)-2 x \mathbf{B}, & {[x J(x)] \partial_{x}=-(\mathfrak{m}-2) J(x)-2 x \mathbf{B},} \\
\partial_{J(x)}[x J(x)]=-(\mathfrak{m}+2) x-2 J(x) \mathbf{B}, & {[x J(x)] \partial_{J(x)}=(\mathfrak{m}-2) x-2 J(x) \mathbf{B} .}\end{cases} \\
& \begin{cases}\partial_{x}\{x, y\}=\{x, y\} \partial_{x}=2 y=\partial_{J(x)}\{J(x), y\}=\{J(x), y\} \partial_{J(x)}, & x \neq y . \\
\partial_{J(x)}\{x, y\}=\{x, y\} \partial_{J(x)}=2 J(y)=-\partial_{x}\{J(x), y\}=-\{J(x), y\} \partial_{x}, & \end{cases}
\end{aligned}
$$

We may now also extend the notion of the directional derivative. For every pair $x \in \mathcal{S}, y \in \mathcal{S} \cup J(\mathcal{S})$, the map $D_{y, x} \in \operatorname{End}(R(\mathcal{S} \cup J(\mathcal{S}), \mathbf{B}))$ is defined by imposing the corresponding extension of the axiom (DD1) and the following version of the axiom (DD2):

$$
\begin{array}{ll}
(\mathbf{D D H} 2) & D_{y, x}[z]=\delta_{x, z} y, \quad D_{y, x}[J(z)]=\delta_{x, z} J(y), \quad z \in \mathcal{S}, \\
& D_{y, x}[\mathbf{B}]=0 .
\end{array}
$$

Note that with this definition we have increased the range of $y$ from $\mathcal{S}$ to $\mathcal{S} \cup J(\mathcal{S})$. This has an important impact on the form of the previous axiom when we are considering $D_{J(y), x}(y \in \mathcal{S})$ :

$$
D_{J(y), x}[z]=\delta_{x, z} J(y), \quad D_{J(y), x}[J(z)]=-\delta_{x, z} y .
$$

The operator $D_{J(y), x}$ is called the twisted directional derivative with respect to $x$ in the direction of $y$.

In [8], the Hermitian radial algebra was introduced using the above abstract complex structure $J$, leading to a definition which is equivalent to the one given in [13]. Let $R_{\mathbb{C}}(\mathcal{S} \cup J(\mathcal{S})$, B) be the complexification of $R(\mathcal{S} \cup J(\mathcal{S}), \mathbf{B})$, i.e.

$$
R_{\mathbb{C}}(\mathcal{S} \cup J(\mathcal{S}), \mathbf{B})=\operatorname{Alg}_{\mathbb{C}}\{\mathcal{S} \cup J(\mathcal{S}) \cup\{\mathbf{B}\}\}=R(\mathcal{S} \cup J(\mathcal{S}), \mathbf{B}) \oplus i R(\mathcal{S} \cup J(\mathcal{S}), \mathbf{B}) .
$$

In this new structure we define the Hermitian conjugation ${ }^{\dagger}$ by

$$
(a+i b)^{\dagger}=\bar{a}-i \bar{b}, \quad a, b \in R(\mathcal{S} \cup J(\mathcal{S}), \mathbf{B}) .
$$

The complex vector variables are introduced as elements of the set:

$$
S_{\mathbb{C}}:=\left\{Z=\frac{1}{2}(x+i J(x)): x \in \mathcal{S}\right\},
$$

together with its Hermitian conjugate

$$
S_{\mathbb{C}}^{\dagger}:=\left\{Z^{\dagger}=-\frac{1}{2}(x-i J(x)): x \in \mathcal{S}\right\} .
$$

These complex vector variables generate, together with $\mathbf{B}$, the Hermitian radial algebra

$$
R\left(S_{\mathbb{C}}, S_{\mathbb{C}}^{\dagger}, \mathbf{B}\right)=\operatorname{Alg}_{\mathbb{C}}\left\{S_{\mathbb{C}} \cup S_{\mathbb{C}}^{\dagger} \cup\{\mathbf{B}\}\right\} \subset R_{\mathbb{C}}(\mathcal{S} \cup J(\mathcal{S}), \mathbf{B})
$$

We can easily check that the Hermitian radial algebra submits to the following rules, which are equivalent to (AH1)-(AH3):

$$
\begin{array}{ll}
\left(\mathbf{A H} 1^{*}\right) & \{Z, U\}=0, \quad\left\{Z^{\dagger}, U^{\dagger}\right\}=0, \quad Z, U \in S_{\mathbb{C}} \\
\left(\mathbf{A H 2}^{*}\right) & {\left[V,\left\{Z, U^{\dagger}\right\}\right]=0, \quad\left[V^{\dagger},\left\{Z, U^{\dagger}\right\}\right]=0, \quad V, Z, U \in S_{\mathbb{C}},} \\
\text { (AH3 } \left.^{*}\right) & {[\mathbf{B}, Z]=2 i Z, \quad\left[\mathbf{B}, Z^{\dagger}\right]=-2 i Z^{\dagger}, \quad Z \in S_{\mathbb{C}} .}
\end{array}
$$

We can also introduce the Hermitian vector derivatives $\partial_{Z}$ and $\partial_{Z^{\dagger}} \in \operatorname{End}\left(R\left(S_{\mathbb{C}}, S_{\mathbb{C}}^{\dagger}, \mathbf{B}\right)\right)$ through the relations

$$
\partial_{Z}:=\frac{1}{4}\left(\partial_{x}-i \partial_{J(x)}\right), \quad \partial_{Z^{\dagger}}:=-\frac{1}{4}\left(\partial_{x}+i \partial_{J(x)}\right)
$$


where $\partial_{x}$ and $\partial_{J(x)}$ are supposed to be linear in the complexification of $R(\mathcal{S} \cup J(\mathcal{S}), \mathbf{B})$. The operators $\partial_{Z}$ and $\partial_{Z^{\dagger}}$ satisfy the following relations which are equivalent to (DH1)-(DH4):

$(\mathrm{DH} 1 *)$

$$
\begin{aligned}
& \partial_{Z}[f F]=\partial_{Z}[f] F+f \partial_{Z}[F], \quad \partial_{Z^{\dagger}}[f F]=\partial_{Z^{\dagger}}[f] F+f \partial_{Z^{\dagger}}[F], \\
& {[f F] \partial_{Z}=F[f] \partial_{Z}+f[F] \partial_{Z}, \quad[f F] \partial_{Z^{\dagger}}=F[f] \partial_{Z^{\dagger}}+f[F] \partial_{Z^{\dagger}},} \\
& f \in R_{0}\left(S_{\mathbb{C}}, S_{\mathbb{C}}^{\dagger}, \mathbf{B}\right):=\operatorname{Alg}_{\mathbb{C}}\left\{\{Z, U\}: Z, U \in S_{\mathbb{C}} \cup S_{\mathbb{C}}^{\dagger}\right\}, \quad F \in R\left(S_{\mathbb{C}}, S_{\mathbb{C}}^{\dagger}, \mathbf{B}\right),
\end{aligned}
$$

$\left(\mathrm{DH}^{*}\right)$

$$
\left\{\begin{array}{l}
\partial_{Z}[G]=[G] \partial_{Z}=0, \\
\partial_{Z}[Z G]=\partial_{Z}[Z] G, \\
{[G Z] \partial_{Z}=G[Z] \partial_{Z}}
\end{array} \quad G \in \operatorname{Alg}_{\mathbb{C}}\left(\left(S_{\mathbb{C}} \backslash\{Z\}\right) \cup S_{\mathbb{C}}^{\dagger} \cup\{\mathbf{B}\}\right),\right.
$$

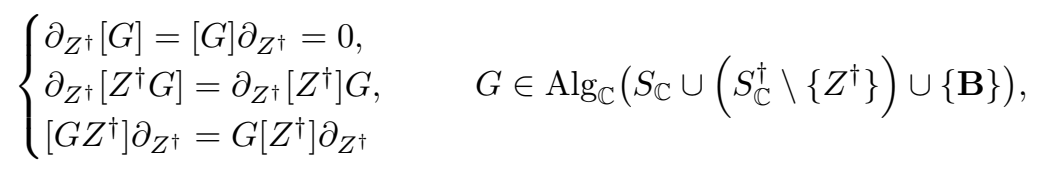

$\left(\mathrm{DH}^{*}\right)$

$$
\begin{aligned}
\partial_{Z}[Z]=\frac{1}{2}(n+i \mathbf{B}), \quad \partial_{Z^{\dagger}}\left[Z^{\dagger}\right]=\frac{1}{2}(n-i \mathbf{B}), \\
{[Z] \partial_{Z}=\frac{1}{2}(n-i \mathbf{B}), \quad\left[Z^{\dagger}\right] \partial_{Z^{\dagger}}=\frac{1}{2}(n+i \mathbf{B}), \quad n=\frac{1}{2} \mathfrak{m}, }
\end{aligned}
$$

$\left(\mathrm{DH} 4^{*}\right)$

$$
\begin{aligned}
& \partial_{Z}(U, Z)=U^{\dagger}=(U, Z) \partial_{Z}, \quad \partial_{Z^{\dagger}}(Z, U)=\partial_{Z^{\dagger}}\left(U^{\dagger}, Z^{\dagger}\right)=U=\left(U^{\dagger}, Z^{\dagger}\right) \partial_{Z^{\dagger}}, \quad Z, U \in S_{\mathbb{C}}, \\
& \text { where }(U, Z):=\left\{U^{\dagger}, Z\right\} .
\end{aligned}
$$

The complex directional derivative and its Hermitian conjugate can then be defined by

$$
D_{U, Z}:=\frac{1}{2}\left(D_{y, x}+i D_{J(y), x}\right), \quad D_{U, Z}^{\dagger}:=\frac{1}{2}\left(D_{y, x}-i D_{J(y), x}\right) .
$$

When $Z=U$ the above operators represent the Hermitian Euler operators $E_{Z}:=D_{Z, Z}$ and $E_{Z^{+}}:=D_{Z, Z}^{\dagger}$.

Some the above endomorphisms abstractly defined on $R(S)$ can be mapped into the algebra of endomorphisms of the Clifford polynomial representation $R\left(\underline{x}_{1}, \ldots, \underline{x}_{s}\right)$. This way, classical Clifford analysis can be seen as a representation of the radial algebra through the following correspondences for each $x \in S:=\left\{x_{1}, \ldots, x_{s}\right\}$

$$
x \rightarrow \underline{x}=\sum_{j=1}^{m} x_{j} e_{j}, \quad x|\rightarrow \underline{x}|=\sum_{j=1}^{m} x_{j} e_{j}\left|, \quad \partial_{x} \rightarrow-\partial_{\underline{x}}=-\sum_{j=1}^{m} e_{j} \partial_{x_{j}}, \quad B \rightarrow-\sum_{j=1}^{m} e_{j} e_{j}\right|,
$$

where the elements $e_{j} \mid: a \rightarrow \widetilde{a} e_{j}$ generate the Clifford algebra $\mathbb{R}_{m, 0}$ and play an important rôle in the isomorphism $\operatorname{End}\left(\mathbb{R}_{0, m}\right) \cong \mathbb{R}_{m, m}$.

The abstract complex structure $J$ can be mapped into the space of linear operators acting on the Clifford-polynomial representation. Observe that the axiom (AH1) implies that in this representation $J \in O(m)$ and $J^{2}=-I_{m}$; which clearly forces the dimension $m$ to be even. This way, $J$ can be mapped to the classical complex structure in the Clifford-polynomial representation with $2 m$ dimensions. Whence Hermitian Clifford analysis can be seen as a representation of the Hermitian radial algebra through the following identifications

$$
\begin{aligned}
& x \rightarrow \underline{x}=\sum_{j=1}^{2 m} x_{j} e_{j}, \quad J(x) \rightarrow J(\underline{x}) \sum_{j=1}^{m}\left(x_{m+j} e_{j}-x_{j} e_{m+j}\right), \quad \mathbf{B} \rightarrow \underline{\mathbf{B}}=\sum_{j=1}^{m} e_{j} e_{m+j}=-i B, \\
& \partial_{x} \rightarrow-\partial_{\underline{x}}=-\sum_{j=1}^{m}\left(e_{j} \partial_{x_{j}}+e_{m+j} \partial_{x_{m+j}}\right), \quad \partial_{J(x)} \rightarrow-\partial_{J(\underline{x})}=-\sum_{j=1}^{m}\left(e_{j} \partial_{x_{m+j}}-e_{m+j} \partial_{x_{j}}\right), \\
& D_{y, x} \rightarrow D_{\underline{y}, \underline{x}}=\sum_{j=1}^{m}\left(y_{j} \partial_{x_{j}}+y_{m+j} \partial_{x_{m+j}}\right), \quad D_{J(y), x} \rightarrow D_{J(\underline{y}), \underline{x}}=\sum_{j=1}^{m}\left(y_{m+j} \partial_{x_{j}}-y_{j} \partial_{x_{m+j}}\right) .
\end{aligned}
$$


For more details about the study of these endomorphisms in these representations we refer the reader to [8]. In the following sections we will introduce the basic objects for defining Hermitian Clifford analysis in superspace through a representation of the Hermitian radial algebra. In order to show that such a definition is consistent we must check that it satisfies the corresponding axioms stated in this section; or equivalently, show that the corresponding map constitutes an algebra homomorphism. This method has been successfully used for the extension of the orthogonal Clifford analysis setting to superspace, see [6].

\section{The superspace framework}

Superanalysis or analysis on superspace considers not only commuting (bosonic) but also anti-commuting (fermionic) variables. In particular these variables can be represented by co-ordinates with values in a graded commutative Banach superalgebra $A=A_{\overline{0}} \oplus A_{\overline{1}}$ over $\mathbb{R}$, where $A_{\overline{0}}$ and $A_{\overline{1}}$ are the spaces of even and odd homogeneous elements respectively and satisfy $A_{\bar{j}} A_{\bar{k}} \subset A_{\overline{j+k}}$ for $\bar{j}, \bar{k} \in \mathbb{Z}_{2}$. We recall that the graded-commutative property means that

$$
v w=w v, \quad v \grave{w}=-w \grave{w}, \quad v w=w v, \quad v, w \in A_{\overline{0}}, \quad \grave{v}, \grave{w} \in A_{\overline{1}} .
$$

An example of such a superalgebra is provided by any Grassmann algebra $\Lambda_{N}=\operatorname{Alg}_{\mathbb{R}}\left\{f_{1}, \ldots, f_{N}\right\}$ where $f_{j} f_{k}+f_{k} f_{j}=0$. For this algebra one has the splitting $\Lambda_{N}=\Lambda_{N}^{+} \oplus \Lambda_{N}^{-}$being $\Lambda_{N}^{+}$the even subspace and $\Lambda_{N}^{-}$the odd subspace. We will not go into detail on the choice of the superalgebra $A$. In order to develop our ideas it just suffices to consider finite sets of commuting and anti-commuting variables.

To establish Clifford analysis in superspace we need to introduce $m$ orthogonal Clifford algebra generators $e_{1}, \ldots, e_{m}$ and $2 n$ symplectic Clifford algebra generators $\grave{e}_{1}, \ldots \grave{e}_{2 n}$, subject to the relations

$$
\begin{aligned}
& e_{j} e_{k}+e_{k} e_{j}=-2 \delta_{j, k} \\
& \grave{e}_{2 j} \grave{e}_{2 k}-\grave{e}_{2 k} \grave{e}_{2 j}=0 \\
& \grave{e}_{2 j-1} \grave{e}_{2 k-1}-\grave{e}_{2 k-1} \grave{e}_{2 j-1}=0 \\
& \grave{e}_{2 j-1}{\grave{e_{2 k}}}-{\grave{e_{2 k}}}_{\hat{e}_{2 j-1}}=\delta_{j, k} \\
& e_{j} \grave{e}_{k}+\grave{e}_{k} e_{j}=0 \text {. }
\end{aligned}
$$

The real algebra generated by both the $e_{j}$ 's and the $\grave{e}_{j}$ 's will be denoted by $\mathcal{C}_{m, 2 n}$.

The classical representation of the radial algebra $R(S)$ in superspace, where $S$ is a finite set composed by $k$ abstract vector variables $(k>1)$, is given by correspondence,

$$
x \rightarrow \mathbf{x}=\underline{x}+\underline{\grave{x}}=\sum_{j=1}^{m} x_{j} e_{j}+\sum_{j=1}^{2 n} \grave{x_{j}} \grave{e_{j}}, \quad x \in S,
$$

between $S$ and the set of independent supervector variables $\mathbf{S}=\{\mathbf{x}: x \in S\}$. For each $x \in S$ we consider in (6) $m$ bosonic (commuting) variables $x_{1}, \ldots, x_{m}$ and $2 n$ fermionic (anti-commuting) variables $\grave{x_{1}}, \ldots, \grave{x_{2 n}}$. The projections $\underline{x}=\sum_{j=1}^{m} x_{j} e_{j}$ and $\underline{\grave{x}}=\sum_{j=1}^{2 n} \grave{x_{j}} \grave{e_{j}}$ are called the bosonic and fermionic vector variables, respectively. The set of independent supervector variables $\mathbf{S}$, obtained through the correspondence (6), generates a radial algebra representation $R(\mathbf{S})$ as we will show next.

Let us define the sets $V A R$ and $V A R$ of bosonic and fermionic variables

$$
V A R=\bigcup_{\mathbf{x} \in \mathbf{S}}\left\{x_{1}, \ldots, x_{m}\right\}, \quad V A R=\bigcup_{\mathbf{x} \in \mathbf{S}}\left\{\grave{x_{1}}, \ldots, \grave{x_{2 n}}\right\}
$$

respectively, where the sets $\left\{x_{1}, \ldots, x_{m}\right\}$ and $\left\{\grave{x_{1}}, \ldots, \grave{x_{2 n}}\right\}$ correspond to the bosonic and fermionic vector variables associated to each $\mathbf{x} \in \mathbf{S}$ through the correspondences (6). This way, $V A R$ contains $m k$ bosonic variables that generate a polynomial algebra $\mathbb{R}[V A R]=\operatorname{Alg}_{\mathbb{R}}\{V A R\}$ while $V A R$ contains $2 n k$ fermionic variables that generate a Grassmann algebra $\Lambda_{2 n k}=\operatorname{Alg}_{\mathbb{R}}\{V A R\}$. They give rise to the algebra of super-polynomials $\mathcal{V}=\mathbb{R}[V A R] \otimes \Lambda_{2 n k}$. The algebra $\mathcal{V}$ is extended to the algebra of Clifford valued super-polynomials

$$
\mathcal{A}_{m, 2 n}=\mathcal{V} \otimes \mathcal{C}_{m, 2 n}
$$


(i.e. the elements of $\mathcal{V}$ commute with the elements of $\mathcal{C}_{m, 2 n}$ ). The correspondence (6) defines a radial algebra representation $\Psi_{m, 2 n}: R(S) \rightarrow \mathcal{A}_{m, 2 n}$, since for every pair $\mathbf{x}, \mathbf{y} \in \mathbf{S}$

$$
\{\mathbf{x}, \mathbf{y}\}=-2 \sum_{j=1}^{m} x_{j} y_{j}+\sum_{j=1}^{n}\left(\grave{x}_{2 j-1} \grave{y}_{2 j}-\grave{x}_{2 j} \grave{y}_{2 j-1}\right)
$$

is a central element in $\mathcal{A}_{m, 2 n}$.

It is important to note that the range $R(\mathbf{S})$ of this representation is a subalgebra strictly contained in $\mathcal{A}_{m, 2 n}$. This can be easily seen by noticing that elements of the form $v \grave{e}_{j}, \grave{v e}_{j}$ with $v \in V A R, \grave{v} \in V A R$ do not belong to $R(\mathbf{S})$. From now on, we will refer to the representation $R(\mathbf{S})$ as the radial algebra embedded in $\mathcal{A}_{m, 2 n}$. Such a representation allows to develop a nice extension of Clifford analysis to superspace as it was shown in $[5,6]$.

Remark 3.1. Let $A=A_{\overline{0}} \oplus A_{\overline{1}}$ be a graded commutative Banach superalgebra. If one considers the variables in $V A R$ and $V A R$ represented as co-ordinates with values in $\Lambda_{\overline{0}}$ and $\Lambda_{\overline{1}}$ respectively; then the corresponding underlying vector space on which the vector variables $\mathbf{x}$ are defined is given by

$$
V=\mathbb{R}^{m \mid 2 n}(A):=\left\{\sum_{j=1}^{m} v_{j} e_{j}+\sum_{j=1}^{2 n} \grave{v_{j}} \grave{e_{j}}: v_{j} \in A_{\overline{0}}, \grave{v_{j}} \in A_{\overline{1}}\right\} .
$$

It is easily seen that the algebra generated by all vectors in $\mathbb{R}^{m \mid 2 n}(A)$ is strictly contained in $A \otimes \mathcal{C}_{m, 2 n}$. In particular, $\mathbb{R}^{m \mid 2 n}(A)$ does not contain the symplectic Clifford generators $\grave{e}_{j}(j=1, \ldots, 2 n)$. This makes the notion of vector variable in this supersymmetry setting more restrictive than in the Clifford-polynomial representation. We recall that in that case, the corresponding underlying vector space is $V=\mathbb{R}^{m}$ which contains all the orthogonal Clifford generators $e_{j}(j=1, \ldots, m)$.

The difference is seen by noticing that the orthogonal Clifford generators satisfy the axiom (A1) since $\left\{e_{j}, e_{k}\right\}$ is always a scalar. But that is not the case for the anti-commutator $\left\{\grave{e}_{j}, \grave{e}_{k}\right\}$ of symplectic Clifford generators. To obtain a representation of the radial algebra in superspace it is necessary to combine the symplectic generators $\grave{e}_{j}$ with anti-commuting variables. In that way we get the commuting element $\left\{\dot{v} \grave{e}_{j}, w \grave{e}_{k}\right\}=\grave{v} w\left[\grave{e}_{j}, \grave{e}_{k}\right]$.

\section{Representation of the radial algebra in superspace}

The endomorphisms defined in section 2 on the radial algebra $R(S)$ can be naturally mapped into the algebra of endomorphisms over $R(\mathbf{S})$. Through this section we will describe the extension of some of these endomorphisms from $R(\mathbf{S})$ to $\mathcal{A}_{m, 2 n}$.

Conjugation: The conjugation admits an extension from the radial algebra representation $R(\mathbf{S})$ to $\mathcal{A}_{m, 2 n}$. In fact, we can define ${ }^{-} \in \operatorname{End}\left(\mathcal{A}_{m, 2 n}\right)$ governed by the following rules:

i) $\div$ is the identity map on $\mathcal{V}$.

ii) $\overline{e_{j_{1}} \cdots e_{j_{k}} \grave{e_{l_{1}}} \cdots \grave{e}_{l_{s}}}=(-1)^{k+\frac{s(s+1)}{2}} \grave{e}_{l_{s}} \cdots \grave{e}_{l_{1}} e_{j_{k}} \cdots e_{j_{1}}$

This extension still is an involution on $\mathcal{A}_{m, 2 n}$ but the anti-automorphism property, i.e. $\overline{F G}=\bar{G} \bar{F}$, which is fulfilled on the radial algebra is not longer satisfied in $\mathcal{A}_{m, 2 n}$. For example, observe that

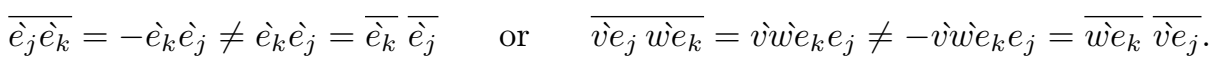

Main involution: The main involution can also be extended from the radial algebra to $\mathcal{A}_{m, 2 n}$. The algebra homomorphism $\sim$ can be defined in a natural way by

i) $\sim$ is the identity map on $\mathcal{V}$.

ii) $\widetilde{e_{j}}=-e_{j}, \quad \widetilde{e_{j}}=-\grave{e}_{j}$.

iii) $\widetilde{F G}=\widetilde{F} \widetilde{G}$. 
Its restricted actions to the bosonic and the fermionic part respectively are called the bosonic and fermionic main involutions and are defined by the following relations:

Bosonic main involution $\sim b$

i) $\sim^{b}$ is the identity map on $\mathcal{V}$,

ii) ${\widetilde{e_{j}}}^{b}=-e_{j}, \quad{\widetilde{e_{j}}}^{b}=\grave{e_{j}}$,

iii) $\widetilde{F G}^{b}=\widetilde{F}^{b} \widetilde{G}^{b}$.
Fermionic main involution $\sim f$

i) $\sim f$ is the identity map on $\mathcal{V}$,

ii) ${\widetilde{e_{j}}}^{f}=e_{j},{\widetilde{e_{j}}}^{f}=-\grave{e}_{j}$,

iii) $\widetilde{F G}^{f}=\widetilde{F}^{f} \widetilde{G}^{f}$.

It is easily seen that the main involution $\sim$ is the composition of its bosonic and fermionic restrictions. There is another important involution in $\mathcal{A}_{m, 2 n}$, related to the fermionic main involution. This algebra homomorphism is called the hash map; it is denoted by $*^{*}$ and defined by the relations:

i) $v^{*}=v$ and $v^{*}=-\grave{v}$ for every $v \in V A R, \grave{v} \in V A R$.

ii) $*^{*}$ is the identity map in $\mathcal{C}_{m, 2 n}$.

iii) $(F G)^{*}=F^{*} G^{*}$.

This hash map will be useful for future computations since for every $F \in \mathcal{A}_{m, 2 n}, \dot{v} \in V A R$ ' we have that $\grave{v} F=F^{*} \grave{v}$ and its restriction to the radial algebra coincides with the fermionic main involution. In fact, for every vector variable $\mathbf{x}$ we have:

$$
\widetilde{\mathbf{x}}^{f}=\sum_{j=1}^{m} x_{j} e_{j}-\sum_{j=1}^{2 n} \grave{x_{j}} \grave{e}_{j}=\mathbf{x}^{*}
$$

Vector derivative: We start by introducing the partial derivatives for every coordinate variable in $V A R \cup V A R$. The left and right derivatives with respect to the commuting variable $v \in V A R$ and the

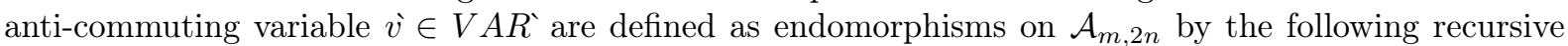
approach:

$$
\left\{\begin{array} { l } 
{ \partial _ { v } [ 1 ] = 0 , } \\
{ \partial _ { v } w - w \partial _ { v } = \delta _ { v , w } , } \\
{ \partial _ { v } \grave { w } = \grave { w } \partial _ { v } , \partial _ { v } e _ { j } = e _ { j } \partial _ { v } , \partial _ { v } \grave { e _ { j } } = \grave { e _ { j } } \partial _ { v } , }
\end{array} \quad \left\{\begin{array}{l}
\partial_{v}[1]=0, \\
\partial_{\grave{v}} \grave{w}+w \partial_{\grave{v}}=\delta_{\grave{v}, w}, \\
\partial_{v} w=w \partial_{\grave{v}}, \partial_{v} e_{j}=e_{j} \partial_{v}, \partial_{v} \grave{e_{j}}=\grave{e_{j}} \partial_{v},
\end{array}\right.\right.
$$

where the above relations also remain valid for the right actions of $w, \dot{w}, \partial_{v}, \partial_{v}$. From this we immediately obtain for $v, w \in V A R$ and $\grave{v}, \grave{w} \in V A R$ that

$$
\partial_{v} \partial_{w}=\partial_{w} \partial_{v}, \quad \partial_{v} \partial_{w}=-\partial_{w} \partial_{v}, \quad \partial_{v} \partial_{w}=\partial_{w} \partial_{v},
$$

which means that the algebra generated by the partial derivatives is isomorphic to $\mathcal{V}$.

It is easily seen that $\partial_{v}, v \in V A R$, behaves like a classical partial derivative. In fact, for $F, G \in \mathcal{A}_{m, 2 n}$ we can prove that

$$
\partial_{v}[F G]=\partial_{v}[F] G+F \partial_{v}[G], \quad[F G] \partial_{v}=F[G] \partial_{v}+[F] \partial_{v} G
$$

By an induction argument, these relations show that the left action of $\partial_{v}$ is equal to its right action if $v \in V A R$, as it is expected. But the situation is no longer the same for the partial derivative $\partial_{v}$ with respect to the anti-commuting variable $v \in V A R$. Consider, for instance, the operator $\partial_{v}$ acting from both sides on the product $\grave{v} \grave{w}$ with $\grave{v} \neq \grave{w}$, i.e.

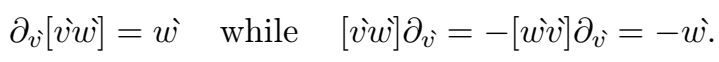

The bosonic Dirac operator $\partial_{\underline{x}}$ and the fermionic Dirac operator $\partial_{\underline{x}}$ associated to the vector variable $\mathbf{x}$ are introduced using the above defined partial derivatives,

$$
\partial_{\underline{x}}=\sum_{j=1}^{m} e_{j} \partial_{x_{j}}, \quad \partial_{\underline{\grave{n}}}=2 \sum_{j=1}^{n}\left(\grave{e_{2 j}} \partial_{\grave{x}_{2 j-1}}-\grave{e_{2 j-1}} \partial_{\grave{x_{2 j}}}\right) .
$$


The left and right super Dirac operators then are defined by

$$
\begin{array}{ll}
\partial_{\mathbf{x}} \cdot=\partial_{\underline{x}} \cdot-\partial_{\underline{x}} \cdot ; \quad F \rightarrow \partial_{\underline{x}}[F]-\partial_{\underline{x}}[F]=\partial_{\mathbf{x}}[F] \\
\cdot \partial_{\mathbf{x}}=-\cdot \partial_{\underline{x}}-\cdot \partial_{\underline{x}} ; \quad F \rightarrow-[F] \partial_{\underline{x}}-[F] \partial_{\underline{x}}=[F] \partial_{\mathbf{x}} .
\end{array}
$$

In [6] it was proven that the above operators satisfy all axioms (D1)- (D4) used in the definition of vector derivative on the level of radial algebra. In this case the rôle of the abstract dimension is played by $\partial_{\mathbf{x}}[\mathbf{x}]=[\mathbf{x}] \partial_{\mathbf{x}}=m-2 n=: M$. This parameter $M$ is called the superdimension.

On the radial algebra level, the left and right actions of the vector derivative are connected by means of the conjugation since $\overline{\partial_{\mathbf{x}}[F]}=-[\bar{F}] \partial_{\mathbf{x}}$ holds for every radial algebra element $F$, see [8]. But this is a property fully dependent on the structure of the radial algebra. That is why the above relation is no longer fulfilled in general on $\mathcal{A}_{m, 2 n}$. For example, we obtain for the element $F={\grave{x_{2 j-1}}}_{\text {that }}$

$$
\overline{\partial_{\mathbf{x}}[F]}=\overline{\partial_{\underline{x}}[F]}=2 \overline{\hat{e}_{2 j}}=-2 \grave{e_{2 j}}, \quad \text { while } \quad-[\bar{F}] \partial_{\mathbf{x}}=[F] \partial_{\underline{x}}=2 \grave{e}_{2 j} .
$$

Vector multipliers: In the Clifford-polynomial representation the vector multipliers $x$ and $x \mid$ can be easily redefined using the basis multipliers $e_{j}$ and $e_{j} \mid$, see (4). In particular, this means that the $\cdot \mid$ action is linear with respect the coordinate variables $x_{j}$. Based on the same idea, we define the following basis multipliers in the superspace representation:

$$
\begin{array}{llll}
e_{j}: & F \rightarrow e_{j} F, & e_{j} \mid: & F \rightarrow \widetilde{F} e_{j}, \\
\grave{e_{j}}: & F \rightarrow \grave{e_{j}} F, & \grave{e_{j}} \mid: & F \rightarrow \widetilde{F}^{b} \grave{e}_{j} .
\end{array}
$$

They allow us to write the $\mathbf{x} \mid$ operator defined on the radial algebra level as

$$
\mathbf{x}|=\underline{x}|+\underline{x}\left|=\sum_{j=1}^{m} x_{j} e_{j}\right|+\sum_{j=1}^{2 n} \grave{x_{j}} \grave{e}_{j} \mid .
$$

In fact, for every $F \in \mathcal{A}_{m, 2 n}$ we have that

$$
\mathbf{x} \mid[F]=\sum_{j=1}^{m} x_{j} \widetilde{F} e_{j}+\sum_{j=1}^{2 n} \grave{x_{j}} \widetilde{F}^{b} \grave{e}_{j}=\sum_{j=1}^{m} \widetilde{F} x_{j} e_{j}+\sum_{j=1}^{2 n}\left(\widetilde{F}^{b}\right)^{*} \grave{x_{j}} \grave{e_{j}} .
$$

Hence, if $F$ is an element of the radial algebra we obtain $\widetilde{F}=\left(\widetilde{F}^{b}\right)^{*}$ and in consequence $\mathbf{x} \mid[F]=\widetilde{F} \mathbf{x}$. Using the identifications:

$$
\begin{array}{cl}
e_{m+j}=i e_{j} \mid & j=1, \ldots, m, \\
\grave{e}_{2 n+j}=i \grave{e}_{j} \mid & j=1, \ldots, 2 n .
\end{array}
$$

it can be easily proven that the operators $e_{1}, \ldots, e_{m}, e_{m+1}, \ldots, e_{2 m}$ and $\grave{e}_{1}, \ldots, \grave{e}_{2 n}, \grave{e}_{2 n+1}, \ldots, \grave{e}_{4 n}$ satisfy the commuting relations given in (5), i.e. they generate the algebra $\mathcal{C}_{2 m, 4 n}$.

For future computations we will need the following relations that can be easily proven using mathematical induction and the recursion formulas (8).

Lemma 1. Let $v \in V A R, \dot{v} \in V A R$ and $\mathbf{x}=\underline{x}+\underline{\grave{x}}$ be a supervector variable. Then one has

$$
\begin{aligned}
& {\left[\partial_{v},{ }^{-}\right]=0, \quad\left[\partial_{v}, \tilde{r}^{-}\right]=0, \quad\left[\partial_{v}, e_{j} \mid\right]=0, \quad\left\{\partial_{\underline{x}}, \sim^{b}\right\}=0, \quad\left[\partial_{\underline{x}}, \sim^{b}\right]=0,} \\
& {\left[\partial_{v}, \sim^{b}\right]=0, \quad\left[\partial_{\dot{v}}, \sim^{b}\right]=0, \quad\left[\partial_{\dot{v}}, e_{j} \mid\right]=0, \quad\left[\partial_{\underline{x}}, \sim^{f}\right]=0, \quad\left\{\partial_{\underline{x}}, \sim^{f}\right\}=0,}
\end{aligned}
$$

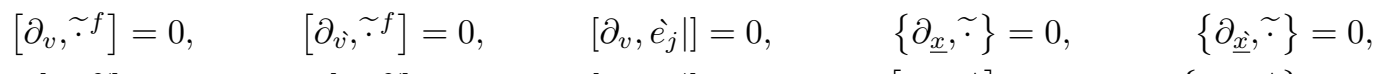

$$
\begin{aligned}
& {\left[\partial_{v}, \widetilde{r}\right]=0, \quad\left[\partial_{\dot{v}}, \widetilde{r}\right]=0, \quad\left[\partial_{\hat{v}}, \grave{e}_{j} \mid\right]=0, \quad\left[\partial_{\underline{x}},{ }^{*}\right]=0, \quad\left\{\partial_{\underline{x}}, \cdot^{*}\right\}=0,} \\
& {\left[\partial_{v}, \cdot^{*}\right]=0, \quad\left\{\partial_{v},{ }^{*}\right\}=0,}
\end{aligned}
$$

on the whole algebra $\mathcal{A}_{m, 2 n}$. In addition, for every $F \in \mathcal{A}_{m, 2 n}$ one has that $\partial_{v}[F]=-\left[F^{*}\right] \partial_{v}$.

Using some of the above properties we can also prove the following differentiation rules 
Lemma 2. Let $v \in V A R, \grave{v} \in V A R$ and $F, G \in \mathcal{A}_{m, 2 n}$. Then one has

$$
\begin{aligned}
& \left\{\begin{array}{l}
\partial_{v}[F G]=\partial_{v}[F] G, \\
{[G F] \partial_{v}=G[F] \partial_{v}, \quad \text { if } G \in A l g_{\mathbb{R}}((V A R \backslash\{v\}) \cup V A R) \otimes \mathcal{C}_{m, 2 n}} \\
\partial_{v}[G]=0=[G] \partial_{v},
\end{array}\right. \\
& \left\{\begin{array}{l}
\partial_{v}[F G]=\partial_{v}[F] G, \\
{[G F] \partial_{v}=G[F] \partial_{v},} \\
\partial_{v}[G]=0=[G] \partial_{v},
\end{array} \quad \text { if } G \in A l g_{\mathbb{R}}(V A R \cup(V A R \backslash\{v\})) \otimes \mathcal{C}_{m, 2 n}\right.
\end{aligned}
$$

Operator B: Following the radial algebra approach and using Lemmas 1 and 2, we can compute the operator $B$ acting on $F \in \mathcal{A}_{m, 2 n}$ as follows:

$$
\begin{aligned}
B[F]= & \left\{\partial_{\mathbf{x}}, \mathbf{x} \mid\right\}[F]=\partial_{\mathbf{x}}[\mathbf{x} \mid[F]]+\mathbf{x} \mid\left[\partial_{\mathbf{x}}[F]\right] \\
= & \left(\partial_{\underline{x}}-\partial_{\underline{x}}\right)(\underline{x}|[F]+\underline{x}|[F])+(\underline{x}|+\underline{x}|)\left(\partial_{\underline{x}}[F]-\partial_{\underline{x}}[F]\right) \\
= & \left(-\partial_{\underline{x}}[\widetilde{F} \underline{x}]+\partial_{\underline{x}}[\widetilde{F}] \underline{x}\right)+\left(-\partial_{\underline{x}}\left[\left(\widetilde{F}^{b}\right)^{*} \underline{x}\right]+\partial_{\underline{x}}\left[\left(\widetilde{F}^{b}\right)^{*}\right] \underline{x}\right)+ \\
& +\left(\partial_{\underline{x}}[\widetilde{F} \underline{x}]-\partial_{\underline{x}}[\widetilde{F}] \underline{x}\right)+\left(\partial_{\underline{x}}\left[\left(\widetilde{F}^{b}\right)^{*} \underline{x}\right]-\partial_{\underline{x}}\left[\left(\widetilde{F}^{b}\right)^{*}\right] \underline{x}\right) \\
= & \left(-\partial_{\underline{x}}[\widetilde{F} \underline{x}]+\partial_{\underline{x}}[\widetilde{F}] \underline{x}\right)+\left(\partial_{\underline{x}}\left[\left(\widetilde{F}^{b}\right)^{*} \underline{x}\right]-\partial_{\underline{x}}\left[\left(\widetilde{F}^{b}\right)^{*}\right] \underline{x}\right) .
\end{aligned}
$$

However

$$
-\partial_{\underline{x}}[\widetilde{F} \underline{x}]+\partial_{\underline{x}}[\widetilde{F}] \underline{x}=-\sum_{j=1}^{m} e_{j} \widetilde{F} e_{j}
$$

and

$$
\begin{aligned}
& \partial_{\underline{\underline{x}}}\left[\left(\widetilde{F}^{b}\right)^{*} \underline{\underline{x}}\right]=2 \sum_{j=1}^{n}\left(\grave{e}_{2 j} \partial_{\grave{x}_{2 j-1}}-\grave{e}_{2 j-1} \partial_{\grave{x}_{2 j}}\right) \sum_{k=1}^{2 n}{\grave{x_{k}}}^{b}{\grave{e_{k}}} \\
& =2 \sum_{\substack{1 \leq j \leq n \\
1 \leq k \leq 2 n}} \grave{e_{2 j}}\left(\delta_{2 j-1, k} \widetilde{F}^{b} \grave{e_{k}}-\grave{x_{k}} \partial_{\grave{x}_{2 j-1}}\left[\widetilde{F}^{b}\right] \grave{e_{k}}\right)-\grave{e_{2 j-1}}\left(\delta_{2 j, k} \widetilde{F}^{b} \grave{e}_{k}-\grave{x_{k}} \partial_{\grave{x}_{2 j}}\left[\widetilde{F}^{b}\right] \grave{e_{k}}\right) \\
& =2 \sum_{\substack{1 \leq j \leq n \\
1 \leq k \leq 2 n}}\left(\delta_{2 j-1, k} \grave{e}_{2 j} \widetilde{F}^{b} \grave{e}_{k}-\delta_{2 j, k} \grave{e}_{2 j-1} \widetilde{F}^{b} \grave{e}_{k}\right)+ \\
& +2 \sum_{\substack{1 \leq j \leq n \\
1 \leq k \leq 2 n}}\left(\grave{e_{2 j}} \partial_{\grave{x}_{2 j-1}}\left[\left(\widetilde{F}^{b}\right)^{*}\right] \grave{x_{k}} \grave{e_{k}}-\grave{e_{2 j-1}} \partial_{\grave{x}_{2 j}}\left[\left(\widetilde{F}^{b}\right)^{*}\right] \grave{x_{k}} \grave{e}_{k}\right)
\end{aligned}
$$

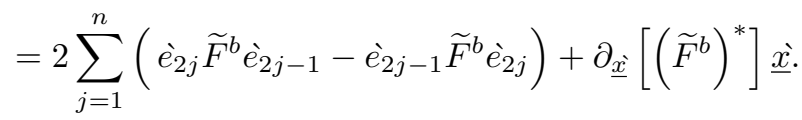

We thus get

$$
B[F]=-\sum_{j=1}^{m} e_{j} \widetilde{F} e_{j}+2 \sum_{j=1}^{n}\left(\grave{e_{2 j}} \widetilde{F}^{b} \grave{e}_{2 j-1}-\grave{e_{2 j-1}} \widetilde{F}^{b} \grave{e}_{2 j}\right),
$$

allowing us to write the operator $B$ as a special "bivector" in $\mathcal{C}_{2 m, 4 n}$ :

$$
B=-\sum_{j=1}^{m} e_{j} e_{j} \mid+2 \sum_{j=1}^{n}\left(\grave{e}_{2 j} \grave{e}_{2 j-1}\left|-\grave{e}_{2 j-1} \grave{e}_{2 j}\right|\right)=i\left(\sum_{j=1}^{m} e_{j} e_{m+j}+2 \sum_{j=1}^{n}\left(\grave{e}_{2 j-1} \grave{e}_{2 n+2 j}-\grave{e}_{2 j} \grave{e}_{2 n+2 j-1}\right)\right) .
$$

In $\mathcal{A}_{m, 2 n}$ we can also define the bosonic and fermionic differential operators

$$
\partial_{\underline{x}}\left|=\sum_{j=1}^{m} e_{j}\right| \partial_{x_{j}}, \quad \partial_{\underline{x}} \mid=2 \sum_{j=1}^{n}\left(\grave{\grave{e}_{2 j}}\left|\partial_{\grave{x}_{2 j-1}}-\grave{e}_{2 j-1}\right| \partial_{\grave{x}_{2 j}}\right)
$$


leading to the super differential operator $\partial_{\mathbf{x}}\left|=\partial_{\underline{x}}\right|-\partial_{\underline{x}} \mid$ acting from the left. Using Lemma 1 we can compute the action of this operator as follows.

$$
\begin{aligned}
\partial_{\mathbf{x}} \mid[F] & =\partial_{\underline{\underline{x}}}\left|[F]-\partial_{\underline{x}}\right|[F]=2 \sum_{j=1}^{n}\left(\grave{e}_{2 j}\left|\partial_{\grave{x}_{2 j-1}}[F]-\grave{\grave{e}_{2 j-1}}\right| \partial_{\grave{x}_{2 j}}[F]\right)-\sum_{j=1}^{m} e_{j} \mid \partial_{x_{j}}[F] \\
& =2 \sum_{j=1}^{n}\left(\partial_{\grave{x}_{2 j-1}}\left[\widetilde{F}^{b}\right]{\grave{e_{2 j}}}-\partial_{\grave{x}_{2 j}}\left[\widetilde{F}^{b}\right] \grave{e}_{2 j-1}\right)-\sum_{j=1}^{m} \partial_{x_{j}}[\widetilde{F}] e_{j} \\
& =-2 \sum_{j=1}^{n}\left(\left[\left(\widetilde{F}^{b}\right)^{*}\right] \partial_{\grave{x}_{2 j-1}} \grave{e}_{2 j}-\left[\left(\widetilde{F}^{b}\right)^{*}\right] \partial_{\grave{x}_{2 j}} \grave{e}_{2 j-1}\right)-\sum_{j=1}^{m}[\widetilde{F}] \partial_{x_{j}} e_{j} \\
& =-\left[\left(\widetilde{F}^{b}\right)^{*}\right] \partial_{\underline{\grave{x}}}-[\widetilde{F}] \partial_{\underline{x}} .
\end{aligned}
$$

Then, on the radial algebra level we have $\partial_{\mathbf{x}} \mid[F]=[\widetilde{F}] \partial_{\mathbf{x}}$ and, as it was proven in $[8]$, the equality $-\left\{\partial_{\mathbf{x}} \mid, \mathbf{x}\right\}=B$ is valid on the radial algebra $R(\mathbf{S})$. By straightforward computation we can check that it remains valid in $\mathcal{A}_{m, 2 n}$.

\section{Complex structures}

Following the approach given in [8], we can define on the radial algebra of doubled vector variables $x=a+i b \mid, a, b \in S$, a complex structure $J_{1}$ by means of

$$
J_{1}(a+i b \mid)=b-i a \mid .
$$

Using the identifications (9), the corresponding version of the doubled vector variables in superspace has the form

$$
\mathbf{x}=\sum_{j=1}^{m} x_{j} e_{j}+\sum_{j=1}^{2 n} \grave{x_{j}} \grave{e}_{j}+i\left(\sum_{j=1}^{m} x_{m+j} e_{j}\left|+\sum_{j=1}^{2 n} \grave{x_{2 n+j}} \grave{e}_{j}\right|\right)=\sum_{j=1}^{2 m} x_{j} e_{j}+\sum_{j=1}^{4 n} \grave{x_{j}} \grave{e}_{j}=\underline{x}+\underline{\grave{x}},
$$

which is the natural form of a vector variable in the algebra $\mathcal{A}_{2 m, 4 n}$. Then the corresponding complex structure defined in the radial algebra embedded in $\mathcal{A}_{2 m, 4 n}$ is given by

$$
J_{1}(\mathbf{x})=J_{1}(\underline{x})+J_{1}(\underline{x})=\sum_{j=1}^{m}\left(x_{m+j} e_{j}-x_{j} e_{m+j}\right)+\sum_{j=1}^{2 n}\left(\grave{x_{2 n+j}} \grave{e_{j}}-\grave{x_{j}} \grave{e}_{2 n+j}\right) .
$$

This radial algebra homomorphism can be extended in a natural way to $\mathcal{A}_{2 m, 4 n}$ by the following relations:

1. $J_{1}$ is the identity map in $\mathcal{V}$.

2. $\quad J_{1}\left(e_{j}\right)=-e_{m+j}, \quad J_{1}\left(e_{m+j}\right)=e_{j}, \quad j=1, \ldots, m$,

$J_{1}\left(\grave{e}_{j}\right)=-\grave{e}_{2 n+j}, \quad J_{1}\left(\grave{e}_{2 n+j}\right)=\grave{e}_{j}, \quad j=1, \ldots, 2 n$.

3. $J_{1}(F G)=J_{1}(F) J_{1}(G), \quad F, G \in \mathcal{A}_{2 m, 4 n}$.

The restriction of $J_{1}$ to the bosonic part $\operatorname{Alg}_{\mathbb{R}}\left(V A R \cup\left\{e_{1}, \ldots, e_{2 m}\right\}\right)$ yields exactly the same complex structure as used in classical Hermitian Clifford analysis (see [2,3]). On the other hand, the restriction to the fermionic part $\operatorname{Alg}_{\mathbb{R}}\left(V A R \cup\left\{\grave{e}_{1}, \ldots, \grave{e}_{4 n}\right\}\right)$ brings new insights to this study since it acts on objects of a different nature.

In the first place observe that the elements $\grave{e}_{j}$ and the algebra generated by them may be represented by polynomial differential operators in $2 n$ dimensions where we introduce commuting variables $a_{j}, b_{j}$ and the corresponding derivatives $\partial_{a_{j}}, \partial_{b_{j}}, j=1, \ldots, n$, and make the assignments (see [19]):

$$
\grave{e}_{2 j-1} \rightarrow \partial_{a_{j}}, \quad \grave{e}_{2 j} \rightarrow a_{j}, \quad \grave{e}_{2 n+2 j-1} \rightarrow \partial_{b_{j}}, \quad \grave{e}_{2 n+2 j} \rightarrow b_{j} .
$$

Indeed, with these identifications we inmediatly obtain the "Weyl algebra defining relations"

$$
\partial_{a_{j}} a_{k}-a_{k} \partial_{a_{j}}=\delta_{j, k}=\partial_{b_{j}} b_{k}-b_{k} \partial_{b_{j}} .
$$


The above approach is completely consistent with the defining relations of the algebra generated only by the $\grave{e}_{j}$ 's. We have to keep in mind that for working with the whole set of Clifford generators $e_{j}$ and $\grave{e}_{j}$ which satisfy the anticommuting relation $e_{j} \grave{e}_{k}=-\grave{e}_{k} e_{j}$, we need to introduce an extra Clifford algebra generator $e_{2 m+1}$ in the previous assignments (see [19])

$$
{\grave{e_{2 j-1}}}_{2 j} e_{2 m+1} \partial_{a_{j}}, \quad{\grave{e_{2 j}}}_{2 j} \rightarrow-e_{2 m+1} a_{j}, \quad \grave{e}_{2 n+2 j-1} \rightarrow e_{2 m+1} \partial_{b_{j}}, \quad \grave{e}_{2 n+2 j} \rightarrow-e_{2 m+1} b_{j} \text {. }
$$

However, this additional element $e_{2 m+1}$ is not important if we are only working with the $\grave{e}_{j}$ 's. The representation (10) is sufficient to study the action of $J_{1}$ over $\operatorname{Alg}_{\mathbb{R}}\left(V A R \cup\left\{\grave{e}_{1}, \ldots, \grave{e}_{4 n}\right\}\right)$.

In the closure of the the set of polynomial differential operators generated by $a_{j}, b_{j}$ we may consider the element

$$
e^{i\langle a, b\rangle}=\exp \left(i \sum_{j=1}^{n} a_{j} b_{j}\right)=\prod_{j=1}^{n} e^{i a_{j} b_{j}}, \quad \text { where } \quad e^{i a_{j} b_{j}}=\sum_{k=0}^{\infty} \frac{\left(i a_{j} b_{j}\right)^{k}}{k !} .
$$

The action of this operator on 1 gives the function $e^{i\langle a, b\rangle}$ that works as a "projection wall" under the action of the above Weyl generators, i.e.

$$
\partial_{a_{j}} e^{i\langle a, b\rangle}[1]=i b_{j} e^{i\langle a, b\rangle}[1], \quad \partial_{b_{j}} e^{i\langle a, b\rangle}[1]=i a_{j} e^{i\langle a, b\rangle}[1]
$$

By means of these relations we obtain a "projection" $J$ of the complex structure $J_{1}$ using the following:

$$
\begin{aligned}
J_{1}\left(\grave{e_{2 j}-1}\right) e^{i\langle a, b\rangle}[1]=-\partial_{b_{j}} e^{i\langle a, b\rangle}[1]=-a_{j} i e^{i\langle a, b\rangle}[1]=-\grave{e}_{2 j} i e^{i\langle a, b\rangle}[1], \quad J\left(\grave{e}_{2 j-1}\right)=-\grave{e}_{2 j}, \\
J_{1}\left(\grave{e_{2 j}}\right) e^{i\langle a, b\rangle}[1]=-b_{j} e^{i\langle a, b\rangle}[1]=\partial_{a_{j}} i e^{i\langle a, b\rangle}[1]=\grave{e}_{2 j-1} i e^{i\langle a, b\rangle}[1], \quad J\left(\grave{e_{2 j}}\right)=\grave{e_{2 j-1}} .
\end{aligned}
$$

In fact, $J$ projects the whole action of $J_{1}$ onto the vector space generated only by the elements $\grave{e}_{1}, \ldots, \grave{e_{2 n}}$. It avoids the "redundancy" caused by doubling the already doubled fermionic part. This shows that in this setting the fermionic dimension does not have to be doubled in order to define a complex structure.

Indeed, the projection $J$ can be extended as an algebra homomorphism of $\mathcal{A}_{2 m, 2 n}$, where only the bosonic dimension has been doubled, using the restriction of $J_{1}$ to the bosonic part. This is:

1. $J$ is the identity in $\mathcal{V}$.

2. $J\left(e_{j}\right)=-e_{m+j}, \quad J\left(e_{m+j}\right)=e_{j}, \quad j=1, \ldots, m$,

2. $J\left(\grave{e}_{2 j-1}\right)=-\grave{e}_{2 j}, \quad J\left(\grave{e}_{2 j}\right)=\grave{e}_{2 j-1}, \quad j=1, \ldots, n$.

3. $J(F G)=J(F) J(G), \quad F, G \in \mathcal{A}_{2 m, 2 n}$.

It is easily seen that $J^{2}(\mathbf{x})=-\mathbf{x}$ for each supervector variable $\mathbf{x}$. This is a natural property for $J$ since this fermionic projection of the complex structure $J_{1}$ also is a complex structure, i.e. it satisfies the complex structure axioms (AH1)-(AH3); as we will show in the next section.

\subsection{Verification of the complex structure axioms}

Here we will check that the action of $J$ on the radial algebra $R(\mathbf{S})$ embedded in $\mathcal{A}_{2 m, 2 n}$ satisfies the complex structure axioms.

In this setting the supervector variables take the form

$$
\mathbf{x}=\underline{x}+\underline{x}=\sum_{j=1}^{m}\left(x_{j} e_{j}+x_{m+j} e_{m+j}\right)+\sum_{j=1}^{n}\left({\grave{x_{2 j-1}}}_{\grave{e}_{2 j-1}}+{\grave{x_{2 j}}}_{2 j}\right),
$$

and the action of $J$ is given by,

$$
J(\mathbf{x})=J(\underline{x})+J(\underline{\hat{x}})=\sum_{j=1}^{m}\left(x_{m+j} e_{j}-x_{j} e_{m+j}\right)+\sum_{j=1}^{n}\left(\grave{x_{2 j}} \grave{e}_{2 j-1}-\grave{x}_{2 j-1} \grave{e}_{2 j}\right) .
$$

\section{Checking (AH1)-(AH2):}

$$
\{\mathbf{x}, \mathbf{y}\}=\{J(\mathbf{x}), J(\mathbf{y})\}, \quad\{J(\mathbf{x}), \mathbf{y}\}=-\{\mathbf{x}, J(\mathbf{y})\} \quad \mathbf{x}, \mathbf{y} \in \mathbf{S}
$$


We clearly have

$$
\begin{aligned}
& \{\underline{x}, \underline{y}\}=-2 \sum_{j=1}^{m}\left(x_{j} y_{j}+x_{m+j} y_{m+j}\right)=\{J(\underline{x}), J(\underline{y})\}, \\
& \{\underline{x}, \underline{y}\}=\sum_{j, k} x_{j} \grave{y_{k}}\left\{e_{j}, \grave{e_{k}}\right\}=0=\{J(\underline{x}), J(\underline{y})\}, \\
& \{\underline{x}, \underline{y}\}=\sum_{j, k} \grave{x_{j}} \grave{y}_{k}\left[\grave{e}_{j}, \grave{e_{k}}\right]=\sum_{j=1}^{n}{\grave{x_{2 j-1}}}_{\grave{y}_{2 j}}-\grave{x_{2 j}} \grave{y}_{2 j-1} \text {. }
\end{aligned}
$$

and also

$$
\begin{aligned}
& \{J(\underline{x}), J(\underline{y})\}=\sum_{j, k=1}^{n}\left\{\grave{x_{2 j}} \grave{e_{2 j-1}}-\grave{x_{2 j-1}} \grave{e_{2 j}}, \grave{y}_{2 k} \grave{e_{2 k-1}}-\grave{y_{2 k-1}} \grave{e_{2 k}}\right\} \\
& =\sum_{j, k=1}^{n}-\grave{x_{2 j}} \grave{y}_{2 k-1}\left[\grave{e}_{2 j-1}, \grave{e_{2 k}}\right]-{\grave{x_{2 j-1}}}_{y_{2 k}}\left[\grave{e}_{2 j}, \grave{e_{2 k-1}}\right] \\
& =\sum_{j=1}^{n}{\grave{x_{2 j-1}}}_{y_{2 j}}-{\grave{x_{2 j}}}_{y_{2 j-1}} \text {. }
\end{aligned}
$$

Hence we conclude,

$$
\{\mathbf{x}, \mathbf{y}\}=-2 \sum_{j=1}^{m}\left(x_{j} y_{j}+x_{m+j} y_{m+j}\right)+\sum_{j=1}^{n}{\grave{x_{2 j-1}}}_{y_{2 j}}-{\grave{x_{2 j}}}_{y_{2 j-1}}=\{J(\mathbf{x}), J(\mathbf{y})\},
$$

which clearly is a central element in $\mathcal{A}_{2 m, 2 n}$.

On the other hand, we have

$$
\begin{aligned}
& \{J(\underline{x}), \underline{y}\}=-2 \sum_{j=1}^{m}\left(x_{m+j} y_{j}-x_{j} y_{m+j}\right)=-\{\underline{x}, J(\underline{y})\}, \\
& \{J(\underline{x}), \underline{y}\}=\sum_{j, k}\left\{x_{m+j} e_{j}-x_{j} e_{m+j}, \grave{y_{k}} \grave{e_{k}}\right\}=0=\{\underline{x}, J(\underline{y})\}, \\
& \{J(\underline{\underline{x}}), \underline{y}\}=\sum_{j, k=1}^{n}\left\{{\grave{x_{2 j}}}_{\grave{e}_{2 j-1}}-{\grave{x_{2 j-1}}}_{\grave{e}_{2 j}},{\grave{y_{2 k-1}}}_{\grave{e}_{2 k-1}}+\grave{y}_{2 k} \grave{e_{2 k}}\right\} \\
& =\sum_{j, k=1}^{n}{\grave{x_{2 j}}}_{y_{2 k}}\left[\grave{e}_{2 j-1},{\grave{e_{2 k}}}\right]-{\grave{x_{2 j-1}}}_{\grave{y}_{2 k-1}}\left[{\grave{e_{2 j}}}, \grave{e}_{2 k-1}\right] \\
& =\sum_{j=1}^{n}{\grave{x_{2 j-1}}}_{\grave{y}_{2 j-1}}+{\grave{x_{2 j}}}_{\grave{y}_{2 j}}
\end{aligned}
$$

and

$$
\begin{aligned}
& \{\underline{\underline{x}}, J(\underline{y})\}=\sum_{j, k=1}^{n}\left\{\grave{x_{2 j-1}} \grave{e_{2 j-1}}+\grave{x_{2 j}} \grave{e_{2 j}}, \grave{y_{2 k}} \grave{e_{2 k-1}}-\grave{y_{2 k-1}} \grave{e_{2 k}}\right\} \\
& =\sum_{j, k=1}^{n}-\grave{x}_{2 j-1} \grave{y}_{2 k-1}\left[\grave{e}_{2 j-1}, \grave{e_{2 k}}\right]+{\grave{x_{2 j}}}_{y_{2 k}}\left[\grave{e}_{2 j}, \grave{e_{2 k-1}}\right] \\
& =-\sum_{j=1}^{n}{\grave{x_{2 j-1}}}_{y_{2 j-1}}+{\grave{x_{2 j}}}_{y_{2 j}} \text {. }
\end{aligned}
$$


Then we obtain

$$
\{J(\mathbf{x}), \mathbf{y}\}=-2 \sum_{j=1}^{m}\left(x_{m+j} y_{j}-x_{j} y_{m+j}\right)+\sum_{j=1}^{n}\left({\grave{x_{2 j-1}}}_{y_{2 j-1}}+\grave{x}_{2 j} \grave{y}_{2 j}\right)=-\{\mathbf{x}, J(\mathbf{y})\},
$$

which also is a central element in $\mathcal{A}_{2 m, 2 n}$.

Checking the axiom (AH3) requires of the introduction of a suitable element $\mathbf{B} \in \mathcal{A}_{2 m, 2 n}$. In accordance with axiom (DH3) we have that such an element $\mathbf{B}$ is obtained by the action of the vector derivative $\partial_{\mathbf{x}}$ on $J(\mathbf{x})$. Then we define,

$$
\begin{aligned}
\mathbf{B} & :=\frac{1}{2} \partial_{\mathbf{x}}[J(\mathbf{x})]=\frac{1}{2}\left(\partial_{\underline{x}}-\partial_{\underline{x}}\right)(J(\underline{x})+J(\underline{x}))=\frac{1}{2}\left(\partial_{\underline{x}}[J(\underline{x})]-\partial_{\underline{x}}[J(\underline{x})]\right)= \\
& =\sum_{j=1}^{m} e_{j} e_{m+j}-\sum_{j=1}^{2 n} \grave{e}_{j}^{2} .
\end{aligned}
$$

This definition is consistent with $(3)$ since $J(\mathbf{B})=\mathbf{B}$.

\section{Checking (AH3):}

$$
[\mathbf{B}, \mathbf{x}]=-2 J(\mathbf{x}), \quad[\mathbf{B}, J(\mathbf{x})]=2 \mathbf{x}, \quad \mathbf{x} \in \mathbf{S} .
$$

Let us write $\mathbf{B}:=\mathbf{B}_{b}-\mathbf{B}_{f}$ with $\mathbf{B}_{b}=\sum_{j=1}^{m} e_{j} e_{m+j}$ and $\mathbf{B}_{f}=\sum_{j=1}^{n}\left({\grave{e_{2 j-1}}}^{2}+\grave{e}_{2 j}{ }^{2}\right)$. We immediately obtain,

$$
\begin{aligned}
& {\left[\mathbf{B}_{b}, \underline{x}\right]=\sum_{j, k} x_{k}\left[e_{j} e_{m+j}, e_{k}\right]=-2 \sum_{j=1}^{m} x_{m+j} e_{j}-x_{j} e_{m+j}=-2 J(\underline{x})} \\
& {\left[\mathbf{B}_{b}, \underline{x}\right]=\sum_{j, k} \grave{x_{k}}\left[e_{j} e_{m+j}, \grave{e_{k}}\right]=0=\sum_{j, k} x_{k}\left[\grave{e}_{j}^{2}, e_{k}\right]=\left[\mathbf{B}_{f}, \underline{x}\right]} \\
& \left.\left.\left[\mathbf{B}_{f}, \underline{x}\right]=\sum_{j, k=1}^{2 n}\left[{\grave{e_{j}}}^{2}, \grave{x}_{k} \grave{e}_{k}\right]=\sum_{j=1}^{n}{\grave{x_{2 k}}}_{\hat{e}_{2 j-1}}{ }^{2}, \grave{e}_{2 k}\right]+{\grave{x_{2 k-1}}}_{\grave{e}_{2 j}}{ }^{2}, \grave{e}_{2 k-1}\right] \\
& =2 \sum_{j=1}^{n}{\grave{x_{2 j}}}_{\grave{e}_{2 j-1}}-{\grave{x_{2 j-1}}}_{\grave{e}_{2 j}}=2 J(\underline{\hat{x}}) \text {. }
\end{aligned}
$$

Then we conclude that

$$
[\mathbf{B}, \mathbf{x}]=\left[\mathbf{B}_{b}, \underline{x}\right]-\left[\mathbf{B}_{f}, \underline{x}\right]=-2 J(\underline{x})-2 J(\underline{x})=-2 J(\mathbf{x}) .
$$

The other equality is obtained by applying $J$ to the above relation.

\subsection{Vector derivatives $\partial_{\mathrm{x}}$ and $\partial_{J(\mathrm{x})}$}

The partial derivatives $\partial_{v}, \partial_{v}$ always commute with the complex structure $J$. Then, the corresponding action of $J$ on the vector derivative $\partial_{\mathbf{x}}$ can be easily seen by means of the following bosonic and fermionic Dirac operators on $\mathcal{A}_{2 m, 2 n}$ :

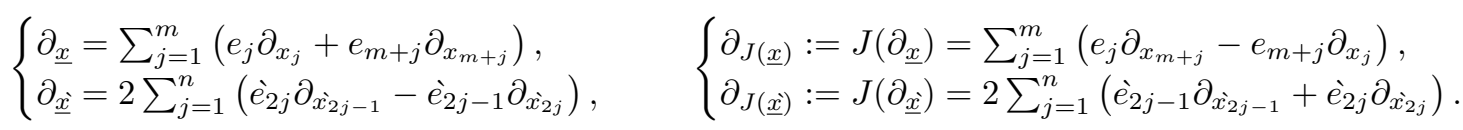

The vector derivatives $\partial_{\mathbf{x}}$ and $\partial_{J(\mathbf{x})}$ then are defined by

$$
\left\{\begin{array}{l}
\partial_{\mathbf{x}} \cdot=\partial_{\underline{\underline{x}}} \cdot-\partial_{\underline{x}} \cdot, \quad\left\{\begin{array}{l}
\partial_{J(\mathbf{x})} \cdot=\partial_{J(\underline{x})} \cdot-\partial_{J(\underline{x})} \cdot \\
\cdot \partial_{\mathbf{x}}=-\cdot \partial_{\underline{x}}-\partial_{\underline{x}},
\end{array} \cdot \partial_{J(\mathbf{x})}=-\cdot \partial_{J(\underline{x})}-\cdot \partial_{J(\underline{x})} .\right.
\end{array}\right.
$$

This means that the above actions are subject to the relations:

$$
\left\{\begin{array} { l } 
{ J ( \partial _ { \mathbf { x } } [ F ] ) = \partial _ { J ( \mathbf { x } } [ J ( F ) ] , } \\
{ J ( [ F ] \partial _ { \mathbf { x } } ) = [ J ( F ) ] \partial _ { J ( \mathbf { x } ) } , }
\end{array} \quad \left\{\begin{array}{l}
J\left(\partial_{J(\mathbf{x})}[F]\right)=-\partial_{\mathbf{x}}[J(F)], \\
J\left([F] \partial_{J(\mathbf{x})}\right)=-[J(F)] \partial_{\mathbf{x}},
\end{array} \quad F \in \mathcal{A}_{2 m, 2 n},\right.\right.
$$


which generalize to $\mathcal{A}_{2 m, 2 n}$ the properties studied at the radial algebra level, see [8].

We now have to verify whether these definitions are in agreement with the axioms (DH1)-(DH4) established in the radial algebra setting. We first check the basic evaluations.

\section{Checking (DH3)}

$$
\begin{array}{r}
\partial_{\mathbf{x}}[\mathbf{x}]=[\mathbf{x}] \partial_{\mathbf{x}}=\mathfrak{m}=\partial_{J(\mathbf{x})}[J(\mathbf{x})]=[J(\mathbf{x})] \partial_{J(\mathbf{x})}, \\
\partial_{\mathbf{x}}[J(\mathbf{x})]=-[J(\mathbf{x})] \partial_{\mathbf{x}}=2 \mathbf{B}=-\partial_{J(\mathbf{x})}[\mathbf{x}]=[\mathbf{x}] \partial_{J(\mathbf{x})} .
\end{array}
$$

It is known from [6] that $\partial_{\mathbf{x}}[\mathbf{x}]=[\mathbf{x}] \partial_{\mathbf{x}}=2 m-2 n=: \mathfrak{m}$, since $\partial_{\mathbf{x}}$ is the original vector derivative in $\mathcal{A}_{2 m, 2 n}$. Then applying $J$ and using (12) we obtain $\partial_{J(\mathbf{x})}[J(\mathbf{x})]=[J(\mathbf{x})] \partial_{J(\mathbf{x})}=2 m-2 n$.

The relation $\partial_{\mathbf{x}}[J(\mathbf{x})]=2 \mathbf{B}$ was the one used in (11). Furthermore,

$$
\begin{aligned}
-[J(\mathbf{x})] \partial_{\mathbf{x}} & =(J(\underline{x})+J(\underline{x}))\left(\partial_{\underline{x}}+\partial_{\underline{x}}\right)=[J(\underline{x})] \partial_{\underline{x}}+[J(\underline{x})] \partial_{\underline{x}} \\
& =2 \sum_{j=1}^{n}\left(-\grave{e}_{2 j-1}{ }^{2}-\grave{e}_{2 j}{ }^{2}\right)+\sum_{j=1}^{m}\left(-e_{m+j} e_{j}+e_{j} e_{m+j}\right) \\
& =2 \mathbf{B} .
\end{aligned}
$$

Applying $J$ on the above equalities and using again (12) we conclude that $-\partial_{J(\mathbf{x})}[\mathbf{x}]=2 \mathbf{B}=[\mathbf{x}] \partial_{J(\mathbf{x})}$.

\section{Checking (DH4)}

$$
\begin{aligned}
& \left\{\begin{array}{ll}
\partial_{\mathbf{x}}\left[\mathbf{x}^{2}\right]=\left[\mathbf{x}^{2}\right] \partial_{\mathbf{x}}=2 \mathbf{x}, & \partial_{J(\mathbf{x})}\left[\mathbf{x}^{2}\right]=\left[\mathbf{x}^{2}\right] \partial_{J(\mathbf{x})}=2 J(\mathbf{x}), \\
\partial_{\mathbf{x}}[\mathbf{x} J(\mathbf{x})]=(\mathfrak{m}+2) J(\mathbf{x})-2 \mathbf{x B}, & {[\mathbf{x} J(\mathbf{x})] \partial_{\mathbf{x}}=-(\mathfrak{m}-2) J(\mathbf{x})-2 \mathbf{x B},} \\
\partial_{J(\mathbf{x})}[\mathbf{x} J(\mathbf{x})]=-(\mathfrak{m}+2) \mathbf{x}-2 J(\mathbf{x}) \mathbf{B}, & {[\mathbf{x} J(\mathbf{x})] \partial_{J(\mathbf{x})}=(\mathfrak{m}-2) \mathbf{x}-2 J(\mathbf{x}) \mathbf{B} .}
\end{array} \quad \mathbf{x} \in \mathbf{S},\right. \\
& \left\{\begin{array}{ll}
\partial_{\mathbf{x}}\{\mathbf{x}, \mathbf{y}\}=\{\mathbf{x}, \mathbf{y}\} \partial_{\mathbf{x}}=2 \mathbf{y}=\partial_{J(\mathbf{x})}\{J(\mathbf{x}), \mathbf{y}\}=\{J(\mathbf{x}), \mathbf{y}\} \partial_{J(\mathbf{x}),} \\
\partial_{J(\mathbf{x})}\{\mathbf{x}, \mathbf{y}\}=\{\mathbf{x}, \mathbf{y}\} \partial_{J(\mathbf{x})}=2 J(\mathbf{y})=-\partial_{\mathbf{x}}\{J(\mathbf{x}), \mathbf{y}\}=-\{J(\mathbf{x}), \mathbf{y}\} \partial_{\mathbf{x}},
\end{array} \quad \mathbf{x} \neq \mathbf{y} .\right.
\end{aligned}
$$

The equalities $\partial_{\mathbf{x}}\left[\mathbf{x}^{2}\right]=\left[\mathbf{x}^{2}\right] \partial_{\mathbf{x}}=2 \mathbf{x}$ and $\partial_{\mathbf{x}}\{\mathbf{x}, \mathbf{y}\}=\{\mathbf{x}, \mathbf{y}\} \partial_{\mathbf{x}}=2 \mathbf{y}(\mathbf{x} \neq \mathbf{y})$ were obtained in [6]. Then, letting act $J$ on each of the previous relations we get,

$$
\partial_{J(\mathbf{x})}\left[\mathbf{x}^{2}\right]=\left[\mathbf{x}^{2}\right] \partial_{J(\mathbf{x})}=2 J(\mathbf{x}), \quad \text { and } \quad \partial_{J(\mathbf{x})}\{\mathbf{x}, \mathbf{y}\}=\{\mathbf{x}, \mathbf{y}\} \partial_{J(\mathbf{x})}=2 J(\mathbf{y}) .
$$

We also find

$$
\begin{aligned}
\partial_{\mathbf{x}}[\mathbf{x} J(\mathbf{x})] & =\left(\partial_{\underline{x}}-\partial_{\underline{x}}\right)(\underline{x}+\underline{x})(J(\underline{x})+J(\underline{\hat{x}})) \\
& =\left(\partial_{\underline{\grave{x}}}-\partial_{\underline{x}}\right)[\underline{x} J(\underline{x})+\underline{x} J(\underline{x})+\underline{\grave{x}} J(\underline{x})+\underline{\grave{x}} J(\underline{\hat{x}})] \\
& =-\partial_{\underline{x}}[J(\underline{x}) \underline{x}]+\partial_{\underline{x}}[\underline{x} J(\underline{x})]+\partial_{\underline{x}}[\underline{x} J(\underline{x})]-\partial_{\underline{x}}[\underline{x} J(\underline{x})]-\partial_{\underline{x}}[\underline{x} J(\underline{x})]+\partial_{\underline{x}}[J(\underline{x}) \underline{x}] .
\end{aligned}
$$

However

$$
\begin{aligned}
& \partial_{\underline{x}}[J(\underline{x}) \underline{x}]=\partial_{\underline{x}}[J(\underline{x})] \underline{x}=-2 \mathbf{B}_{f} \underline{x}, \\
& \partial_{\underline{x}}[\underline{x} J(\underline{x})]=\partial_{\underline{x}}[\underline{x}] J(\underline{x})=-2 n J(\underline{x}), \\
& \partial_{\underline{x}}[\underline{x} J(\underline{x})]=\partial_{\underline{x}}[\underline{x}] J(\underline{x})=-2 m J(\underline{x}), \\
& \partial_{\underline{x}}[J(\underline{x}) \underline{x}]=\partial_{\underline{x}}[J(\underline{x})] \underline{\grave{x}}=-2 \mathbf{B}_{b} \underline{x}, \\
& \partial_{\underline{x}}[\underline{\grave{x}} J(\underline{\grave{x}})]=2 \sum_{j=1}^{n} \grave{e}_{2 j} \partial_{\grave{x}_{2 j-1}}[\underline{\grave{x}} J(\underline{\grave{x}})]-\grave{e}_{2 j-1} \partial_{\grave{x}_{2 j}}[\underline{\grave{x}} J(\underline{x})]
\end{aligned}
$$

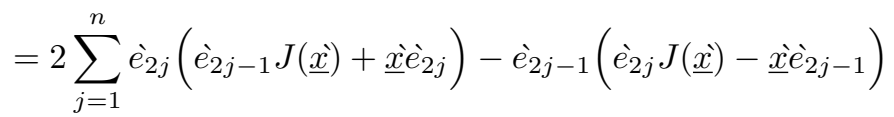

$$
\begin{aligned}
& =2\left(\sum_{j=1}^{n} \grave{e}_{2 j} \grave{e}_{2 j-1}-\grave{e}_{2 j-1} \grave{e_{2 j}}\right) J(\underline{x})+2 \sum_{j=1}^{n}\left(\grave{\grave{e}_{2 j-1}} \underline{x}_{\grave{e}} \grave{e}_{2 j-1}+\grave{e}_{2 j} \underline{\underline{x} \grave{e}_{2 j}}\right) \\
& =-2 n J(\underline{x})+2 \sum_{j=1}^{n}\left({\grave{e_{2 j-1}}}^{2} \underline{\grave{x}}-\grave{e}_{2 j-1}{\grave{x_{2 j}}}+\grave{e}_{2 j}{ }^{2} \underline{\grave{x}}+{\grave{e_{2 j}}}_{\hat{x}_{2 j-1}}\right) \\
& =-(2 n+2) J(\underline{x})+2 \mathbf{B}_{f} \underline{\grave{x}}
\end{aligned}
$$


and

$$
\begin{aligned}
\partial_{\underline{x}}[\underline{x} J(\underline{x})] & =\sum_{j=1}^{m} e_{j}\left(e_{j} J(\underline{x})-\underline{x} e_{m+j}\right)+e_{m+j}\left(e_{m+j} J(\underline{x})+\underline{x} e_{j}\right) \\
& =-2 m J(\underline{x})+\sum_{j=1}^{m}\left(e_{m+j} \underline{x} e_{j}-e_{j} \underline{x} e_{m+j}\right) \\
& =-2 m J(\underline{x})+\sum_{j=1}^{m}\left(2 e_{j} e_{m+j} \underline{x}+2 x_{m+j} e_{j}-2 x_{j} e_{m+j}\right) \\
& =(-2 m+2) J(\underline{x})+2 \mathbf{B}_{b} \underline{x} .
\end{aligned}
$$

Then we conclude that

$$
\begin{aligned}
\partial_{\mathbf{x}}[\mathbf{x} J(\mathbf{x})] & =2 \mathbf{B}_{f} \underline{x}-2 n J(\underline{x})-(2 n+2) J(\underline{x})+2 \mathbf{B}_{f} \underline{\grave{x}}-(-2 m+2) J(\underline{x})-2 \mathbf{B}_{b} \underline{x}+2 m J(\underline{\grave{x}})-2 \mathbf{B}_{b} \underline{\grave{x}} \\
& =\left(2 \mathbf{B}_{f}-2 \mathbf{B}_{b}\right) \underline{x}+\left(2 \mathbf{B}_{f}-2 \mathbf{B}_{b}\right) \underline{\grave{x}}+(2 m-2 n-2) J(\underline{x})+(2 m-2 n-2) J(\underline{x}) \\
& =(2 m-2 n-2) J(\mathbf{x})-2 \mathbf{B} \mathbf{x} \\
& =(2 m-2 n+2) J(\mathbf{x})-2 \mathbf{x B} .
\end{aligned}
$$

Letting act $J$ on (13) we obtain:

$$
\partial_{J(\mathbf{x})}[\mathbf{x} J(\mathbf{x})]=-(\mathfrak{m}+2) \mathbf{x}-2 J(\mathbf{x}) \mathbf{B},
$$

and conjugating both (13) and (14), we get

$$
[\mathbf{x} J(\mathbf{x})] \partial_{\mathbf{x}}=-(\mathfrak{m}-2) J(\mathbf{x})-2 \mathbf{x B}, \quad \text { and } \quad[\mathbf{x} J(\mathbf{x})] \partial_{J(\mathbf{x})}=(\mathfrak{m}-2) \mathbf{x}-2 J(\mathbf{x}) \mathbf{B} .
$$

Furthermore we have

$$
\begin{aligned}
& \partial_{\mathbf{x}}\{J(\mathbf{x}), \mathbf{y}\}=\left(\partial_{\underline{\grave{x}}}-\partial_{\underline{x}}\right)\left(-2 \sum_{j=1}^{m}\left(x_{m+j} y_{j}-x_{j} y_{m+j}\right)+\sum_{j=1}^{n}\left(\grave{x_{2 j-1}}{\grave{y_{2 j-1}}}+\grave{x_{2 j} \grave{y_{2 j}}}\right)\right) \\
& =2 \sum_{j=1}^{m}\left(e_{m+j} y_{j}-e_{j} y_{m+j}\right)+2 \sum_{j=1}^{n}\left(\grave{e}_{2 j} \grave{y}_{2 j-1}-\grave{e}_{2 j-1} \grave{y}_{2 j}\right) \\
& =-2 J(\underline{y})-2 J(\underline{y})=-2 J(\mathbf{y}) \\
& =\left(-2 \sum_{j=1}^{m}\left(x_{m+j} y_{j}-x_{j} y_{m+j}\right)+\sum_{j=1}^{n}\left({\grave{x_{2 j-1}}}_{\grave{y}_{2 j-1}}+{\grave{x_{2 j}}}_{y_{2 j}}\right)\right)\left(-\partial_{\underline{x}}-\partial_{\underline{x}}\right) \\
& =\{J(\mathbf{x}), \mathbf{y}\} \partial_{\mathbf{x}} .
\end{aligned}
$$

Finally, from the action of $J$ on the above relations we obtain

$$
\partial_{J(\mathbf{x})}\{J(\mathbf{x}), \mathbf{y}\}=2 \mathbf{y}=\{J(\mathbf{x}), \mathbf{y}\} \partial_{J(\mathbf{x})} .
$$

\section{Checking (DH1)}

$$
\begin{aligned}
& \partial_{\mathbf{x}}[f F]=\partial_{\mathbf{x}}[f] F+f \partial_{\mathbf{x}}[F], \quad \partial_{J(\mathbf{x})}[f F]=\partial_{J(\mathbf{x})}[f] F+f \partial_{J(\mathbf{x})}[F], \\
& {[f F] \partial_{\mathbf{x}}=F[f] \partial_{\mathbf{x}}+f[F] \partial_{\mathbf{x}}, \quad[f F] \partial_{J(\mathbf{x})}=F[f] \partial_{J(\mathbf{x})}+f[F] \partial_{J(\mathbf{x})},} \\
& f \in R_{0}(\mathbf{S} \cup J(\mathbf{S})), \quad F \in R(\mathbf{S} \cup J(\mathbf{S}), \mathbf{B}) .
\end{aligned}
$$

It suffices to prove (DH1) just for those $f$ generating $R_{0}(\mathbf{S} \cup J(\mathbf{S})$ ), which are given by the anticommutators

$$
\{\mathbf{x}, \mathbf{y}\}, \quad \mathbf{x}^{2}, \quad\{J(\mathbf{x}), \mathbf{y}\}, \quad \mathbf{x}, \mathbf{y} \in \mathbf{S} .
$$


By straightforward computation we obtain for every $F \in \mathcal{A}_{2 m, 2 n}$ that

$$
\left\{\begin{array} { l l } 
{ \partial _ { \mathbf { x } } [ \{ \mathbf { x } , \mathbf { y } \} F ] = 2 \mathbf { y } F + \{ \mathbf { x } , \mathbf { y } \} \partial _ { \mathbf { x } } [ F ] , } \\
{ \partial _ { \mathbf { x } } [ \mathbf { x } ^ { 2 } F ] = 2 \mathbf { x } F + \mathbf { x } ^ { 2 } \partial _ { \mathbf { x } } [ F ] , } \\
{ \partial _ { \mathbf { x } } [ \{ J ( \mathbf { x } ) , \mathbf { y } \} F ] = - 2 J ( \mathbf { y } ) F + \{ J ( \mathbf { x } ) , \mathbf { y } \} \partial _ { \mathbf { x } } [ F ] , }
\end{array} \quad \left\{\begin{array}{l}
{[\{\mathbf{x}, \mathbf{y}\} F] \partial_{\mathbf{x}}=2 F \mathbf{y}+\{\mathbf{x}, \mathbf{y}\}[F] \partial_{\mathbf{x}},} \\
{\left[\mathbf{x}^{2} F\right] \partial_{\mathbf{x}}=2 F \mathbf{x}+\mathbf{x}^{2}[F] \partial_{\mathbf{x}},} \\
{[\{J(\mathbf{x}), \mathbf{y}\} F] \partial_{\mathbf{x}}=-2 F J(\mathbf{y})+\{J(\mathbf{x}), \mathbf{y}\}[F] \partial_{\mathbf{x}} .}
\end{array}\right.\right.
$$

Finally, the other relations can be obtained by means of the action of $J$ on the above equality.

The statement in (DH2) being a trivial consequence of Lemma 2, we omit its proof.

\subsection{Directional derivatives}

We are now able to obtain explicit expressions for the directional derivatives $D_{\mathbf{y}, \mathbf{x}}$ and $D_{J(\mathbf{y}), \mathbf{x}}$ in this setting. From the radial algebra framework (see [8]) it is known that

$$
\left\{\partial_{\mathbf{x}}, \mathbf{y}\right\}=2 D_{\mathbf{y}, \mathbf{x}}+\delta_{\mathbf{x}, \mathbf{y}} \mathfrak{m}, \quad\left\{\partial_{\mathbf{x}}, J(\mathbf{y})\right\}=2 D_{J(\mathbf{y}), \mathbf{x}}+2 \delta_{\mathbf{x}, \mathbf{y}} \mathbf{B} .
$$

For the operator $\left\{\partial_{\mathbf{x}}, \mathbf{y}\right\}$ we first obtain

$$
\begin{aligned}
& \left\{\partial_{\mathbf{x}}, \mathbf{y}\right\}=\left\{-\sum_{j=1}^{2 m} e_{j} \partial_{x_{j}}+2 \sum_{j=1}^{n}\left(\grave{e_{2 j}} \partial_{\grave{x}_{2 j-1}}-\grave{e}_{2 j-1} \partial_{\grave{x}_{2 j}}\right), \sum_{k=1}^{2 m} y_{k} e_{k}+\sum_{k=1}^{2 n} \grave{y_{k}} \grave{e_{k}}\right\} \\
& =-\sum_{j, k=1}^{2 m}\left\{e_{j} \partial_{x_{j}}, y_{k} e_{k}\right\}-\sum_{\substack{1 \leq j \leq 2 m \\
1 \leq k \leq 2 n}}\left\{e_{j} \partial_{x_{j}}, \grave{y_{k}} \grave{e_{k}}\right\}+2 \sum_{\substack{1 \leq j \leq n \\
1 \leq k \leq 2 m}}\left(\left\{\grave{\grave{e}_{2 j}} \partial_{\grave{x}_{2 j-1}}, y_{k} e_{k}\right\}-\left\{\grave{e_{2 j-1}} \partial_{\grave{x}_{2 j}}, y_{k} e_{k}\right\}\right) \\
& +2 \sum_{\substack{1 \leq j \leq n \\
1 \leq k \leq 2 n}}\left(\left\{\grave{e}_{2 j} \partial_{\grave{x}_{2 j-1}}, \grave{y}_{k} \grave{e}_{k}\right\}-\left\{\grave{e}_{2 j-1} \partial_{\grave{x}_{2 j}}, \grave{y_{k}} \grave{e}_{k}\right\}\right) .
\end{aligned}
$$

However, from (5) and (8), we obtain the following relations for every pair $v, w \in V A R$ and every pair $\grave{v}, \grave{w} \in V A R:$

$$
\begin{aligned}
& \left\{e_{j} \partial_{v}, e_{k} w\right\}=-\delta_{v, w} e_{k} e_{j}-2 \delta_{j, k} \delta_{v, w}-2 \delta_{j, k} w \partial_{v}, \\
& \left\{e_{j} \partial_{v}, \grave{e}_{k} v\right\}=0=\left\{\grave{e}_{j} \partial_{\grave{v}}, e_{k} v\right\} \text {, } \\
& \left\{\grave{e}_{2 j} \partial_{v}, \grave{e}_{k} w\right\}=\delta_{\dot{v}, w} \grave{e}_{k} \grave{e}_{2 j}-\delta_{2 j-1, k} \delta_{v, w}+\delta_{2 j-1, k} w \partial_{v}, \\
& \left\{\grave{e}_{2 j-1} \partial_{v}, \grave{e}_{k} w\right\}=\delta_{v, w} \grave{e}_{k} \grave{e}_{2 j-1}+\delta_{2 j, k} \delta_{\grave{v}, w}-\delta_{2 j, k} w \partial_{v} \text {, }
\end{aligned}
$$

whence,

$$
\begin{aligned}
\left\{\partial_{\mathbf{x}}, \mathbf{y}\right\}= & -\sum_{j, k=1}^{2 m}\left(-\delta_{\mathbf{x}, \mathbf{y}} \delta_{j, k} e_{k} e_{j}-2 \delta_{j, k} \delta_{\mathbf{x}, \mathbf{y}}-2 \delta_{j, k} y_{k} \partial_{x_{j}}\right) \\
& +2 \sum_{\substack{1 \leq j \leq n \\
1 \leq k \leq 2 n}}\left(\delta_{\mathbf{x}, \mathbf{y}} \delta_{2 j-1, k} \grave{e_{k} \grave{e}_{2 j}}-\delta_{2 j-1, k} \delta_{\mathbf{x}, \mathbf{y}}+\delta_{2 j-1, k} \grave{y_{k}} \partial_{\grave{x}_{2 j-1}}\right) \\
& -2 \sum_{\substack{1 \leq j \leq n \\
1 \leq k \leq 2 n}}\left(\delta_{\mathbf{x}, \mathbf{y}} \delta_{2 j, k} \grave{e_{k}} \grave{e}_{2 j-1}+\delta_{2 j, k} \delta_{\mathbf{x}, \mathbf{y}}-\delta_{2 j, k} \grave{y_{k}} \partial_{\grave{x}_{2 j}}\right) \\
= & \sum_{j=1}^{2 m}\left(\delta_{\mathbf{x}, \mathbf{y}}+2 y_{j} \partial_{x_{j}}\right)+2 \sum_{j=1}^{n}\left(\delta_{\mathbf{x}, \mathbf{y}}\left(\grave{e}_{2 j-1} \grave{e}_{2 j}-\grave{e}_{2 j} \grave{e}_{2 j-1}-2\right)+\grave{y}_{2 j-1} \partial_{\grave{x}_{2 j-1}}+\grave{y}_{2 j} \partial_{\grave{x}_{2 j}}\right) \\
= & \delta_{\mathbf{x}, \mathbf{y}}(2 m-2 n)+2\left(\sum_{j=1}^{2 m} y_{j} \partial_{x_{j}}+\sum_{j=1}^{2 n} \grave{y_{j}} \partial_{\grave{x_{j}}}\right) .
\end{aligned}
$$


Similarly, for the operator $\left\{\partial_{\mathbf{x}}, J(\mathbf{y})\right\}$ we obtain

$$
\begin{aligned}
& \left\{\partial_{\mathbf{x}}, J(\mathbf{y})\right\}=\left\{\partial_{\underline{x}}-\partial_{\underline{x}}, \sum_{k=1}^{2 m}\left(y_{m+k} e_{k}-y_{k} e_{m+k}\right)+\sum_{k=1}^{2 n}\left(\grave{y}_{2 k} \grave{e}_{2 k-1}-\grave{y}_{2 k-1} \grave{e}_{2 k}\right)\right\} \\
& =-\sum_{j, k=1}^{m}\left\{e_{j} \partial_{x_{j}}, e_{k} y_{m+k}\right\}-\left\{e_{j} \partial_{x_{j}}, e_{m+k} y_{k}\right\}+\left\{e_{m+j} \partial_{x_{m+j}}, e_{k} y_{m+k}\right\}-\left\{e_{m+j} \partial_{x_{m+j}}, e_{m+k} y_{k}\right\} \\
& +2 \sum_{j, k=1}^{n}\left\{\grave{e}_{2 j} \partial_{\grave{x}_{2 j-1}}, \grave{e}_{2 k-1} \grave{y_{2 k}}\right\}-\left\{\grave{e}_{2 j} \partial_{\grave{x}_{2 j-1}}, \grave{e}_{2 k} \grave{y_{2 k}}\right\}-\left\{\grave{e}_{2 j-1} \partial_{\grave{x}_{2 j}}, \grave{e}_{2 k-1} \grave{y}_{2 k}\right\}+\left\{\grave{e}_{2 j-1} \partial_{\grave{x}_{2 j}}, \grave{e}_{2 k} \grave{2}_{2 k-1}\right\} \\
& =-\sum_{j, k=1}^{m}\left(-2 \delta_{j, k} y_{m+k} \partial_{x_{j}}+\delta_{\mathbf{x}, \mathbf{y}} \delta_{j, k} e_{m+k} e_{j}-\delta_{\mathbf{x}, \mathbf{y}} \delta_{j, k} e_{k} e_{m+j}+2 \delta_{j, k} y_{k} \partial_{x_{m+j}}\right) \\
& +2 \sum_{j, k=1}^{n}\left(\delta_{j, k} \grave{y_{2 k}} \partial_{\grave{x}_{2 j-1}}-\delta_{\mathbf{x}, \mathbf{y}} \delta_{j, k} \grave{e_{2 k} \grave{e_{2 j}}}-\delta_{\mathbf{x}, \mathbf{y}} \delta_{j, k} \grave{e_{2 k-1}} \grave{e}_{2 j-1}-\delta_{j, k} \grave{y_{2 k-1}} \partial_{\grave{x}_{2 k}}\right) \\
& =2 \sum_{j=1}^{m}\left(\delta_{\mathbf{x}, \mathbf{y}} e_{j} e_{m+j}+y_{m+j} \partial_{x_{j}}-y_{j} \partial_{m+j}\right)+2 \sum_{j=1}^{n}\left(-\delta_{\mathbf{x}, \mathbf{y}}\left(\grave{e}_{2 j-1}{ }^{2}+\grave{e}_{2 j}{ }^{2}\right)+\grave{y}_{2 j} \partial_{\grave{x}_{2 j-1}}-\grave{y}_{2 j-1} \partial_{\grave{x}_{2 j}}\right) \\
& =2 \delta_{\mathbf{x}, \mathbf{y}} \mathbf{B}+2\left(\sum_{j=1}^{m}\left(y_{m+j} \partial_{x_{j}}-y_{j} \partial_{x_{m+j}}\right)+\sum_{j=1}^{n}\left(\grave{y}_{2 j} \partial_{\grave{x}_{2 j-1}}-\grave{y}_{2 j-1} \partial_{\grave{x}_{2 j}}\right)\right) .
\end{aligned}
$$

Then, we have found the following expresions for the directional derivatives

$$
\begin{aligned}
D_{\mathbf{y}, \mathbf{x}} & =D_{\underline{y}, \underline{x}}+D_{\underline{\underline{y}}, \underline{\underline{x}}}=\sum_{j=1}^{2 m} y_{j} \partial_{x_{j}}+\sum_{j=1}^{2 n} \grave{y_{j}} \partial_{\grave{x_{j}}}, \\
D_{J(\mathbf{y}), \mathbf{x}} & =D_{J(\underline{y}), \underline{x}}+D_{J(\underline{y}), \underline{x}}=\sum_{j=1}^{m}\left(y_{m+j} \partial_{x_{j}}-y_{j} \partial_{x_{m+j}}\right)+\sum_{j=1}^{n}\left(\grave{y_{2 j}} \partial_{\grave{x_{2 j-1}}}-\grave{y_{2 j-1}} \partial_{\grave{x}_{2 j}}\right),
\end{aligned}
$$

which lead to the Euler operator $\mathbb{E}_{\mathbf{x}}=D_{\mathbf{x}, \mathbf{x}}=\sum_{j=1}^{2 m} x_{j} \partial_{x_{j}}+\sum_{j=1}^{2 n} \grave{x_{j}} \partial_{\grave{x_{j}}}$. As it is known, $\mathbb{E}_{\mathbf{x}}$ measures the degree of homogeneity in the supervector variable $\mathbf{x}$ of every element of the radial algebra embedded in $\mathcal{A}_{2 m, 2 n}$. This situation can be generalized to the polynomials setting.

Let $\mathcal{P}:=\mathbb{C}\left[x_{1}, \ldots, x_{2 m}, \grave{x_{1}}, \ldots, \grave{x_{2 n}}\right]$ be the space of complex valued polynomials in the variables $x_{1}, \ldots, x_{2 m}, \grave{x_{1}}, \ldots, \grave{x_{2 n}}$. As in the classical framework, a polynomial $R_{k}(x)=R_{k}\left(x_{1}, \ldots, x_{2 m}, \grave{x_{1}}, \ldots, \grave{x_{2 n}}\right)$ is said to be homogenoeus of degree $k \in \mathbb{N}$ if for every $\lambda \in \mathbb{C} \backslash\{0\}$ it holds that

$$
R_{k}(\lambda x)=\lambda^{k} R_{k}(x) .
$$

The complex vector space of homogeneous polynomials of degree $k$ in $\mathcal{P}$ is denoted by $\mathcal{P}_{k}$. A basis for $\mathcal{P}_{k}$ consist of elements of the form $x_{1}^{\alpha_{1}} \cdots x_{2 m}^{\alpha_{2 m}} x_{1}^{\beta_{1}} \cdots x_{2 n}^{\beta_{2 n}}$ where $\alpha_{j} \in \mathbb{N}, \beta_{j} \in\{0,1\}$ and with $\sum_{j=1}^{2 m} \alpha_{j}+\sum_{j=1}^{2 n} \beta_{j}=k$. It is moreover easily seen that $\mathcal{P}_{k}$ is a finite dimensional vector space with dimension

$$
\operatorname{dim} \mathcal{P}_{k}=\sum_{j=0}^{\min (k, 2 n)}\left(\begin{array}{c}
2 n \\
j
\end{array}\right)\left(\begin{array}{c}
k-j+2 m-1 \\
2 m-1
\end{array}\right) .
$$

It can be directly verified that $\mathcal{P}_{k}$ is an eigenspace of $\mathbb{E}_{x}$ with eigenvalue $k$. The same conclusion holds for $\mathcal{P}_{k} \otimes \mathcal{C}_{2 m, 2 n}$.

\section{Hermitian setting in superspace}

In the complexification $\mathbb{C} \mathcal{A}_{2 m, 2 n}$ of $\mathcal{A}_{2 m, 2 n}$ we define the Hermitian conjugation . $\dagger$ as

$$
(a+i b)^{\dagger}=\bar{a}-i \bar{b}, \quad a, b \in \mathcal{A}_{2 m, 2 n},
$$

which generalizes the Hermitian conjugation over the complexification of the radial algebra with the complex structure introduced in Section 2, see also [8]. 
The Hermitian vector variables in superspace are then introduced by

$$
\begin{aligned}
z & =\frac{1}{2}(\mathbf{x}+i J(\mathbf{x}))=\frac{1}{2}(\underline{x}+i J(\underline{x}))+\frac{1}{2}(\underline{x}+i J(\underline{x}))=: \underline{z}+\underline{z}, \\
z^{\dagger} & =-\frac{1}{2}(\mathbf{x}-i J(\mathbf{x}))=-\frac{1}{2}(\underline{x}-i J(\underline{x}))-\frac{1}{2}(\underline{\grave{x}}-i J(\underline{x}))=: \underline{z}^{\dagger}+\underline{z}^{\dagger},
\end{aligned}
$$

defining the bosonic Hermitian vector variables $\underline{z}, \underline{z}^{\dagger}$ and the fermionic Hermitian vector variables $\underline{z}, \underline{z}^{\dagger}$ as follows

$$
\begin{aligned}
& \underline{z}=\frac{1}{2}(\underline{x}+i J(\underline{x}))=\frac{1}{2} \sum_{j=1}^{m}\left(x_{j}+i x_{m+j}\right)\left(e_{j}-i e_{m+j}\right)=\sum_{j=1}^{m} z_{j} \mathfrak{f}_{j}, \\
& \underline{z}^{\dagger}=-\frac{1}{2}(\underline{x}-i J(\underline{x}))=-\frac{1}{2} \sum_{j=1}^{m}\left(x_{j}-i x_{m+j}\right)\left(e_{j}+i e_{m+j}\right)=\sum_{j=1}^{m} z_{j}^{c} \mathfrak{f}_{j}^{\dagger}, \\
& \underline{z}=\frac{1}{2}(\underline{\underline{x}}+i J(\underline{x}))=\frac{1}{2} \sum_{j=1}^{n}\left(\grave{x_{2 j-1}}+i \grave{x_{2 j}}\right)\left(\grave{e_{2 j-1}}-i \grave{e_{2 j}}\right)=\sum_{j=1}^{n} \grave{z_{j}} \mathfrak{f}_{j}, \\
& \underline{\underline{z}}^{\dagger}=-\frac{1}{2}(\underline{\grave{x}}-i J(\underline{x}))=-\frac{1}{2} \sum_{j=1}^{n}\left(\grave{x_{2 j-1}}-i \grave{x_{2 j}}\right)\left(\grave{e_{2 j-1}}+i \grave{e_{2 j}}\right)=\sum_{j=1}^{n} z_{j}^{c} \mathfrak{f}_{j}^{\dagger \dagger} .
\end{aligned}
$$

Here we have introduced the commuting complex variables $z_{j}=x_{j}+i x_{m+j}$, the anti-commuting variables $\grave{z_{j}}={\grave{x_{2 j-1}}}+\grave{x_{2 j}}$, their complex conjugates $z_{j}^{c}=x_{j}-i x_{m+j}, z_{j}^{c}={\grave{x_{2 j-1}}}-\grave{x_{2 j}}$ and the Witt basis elements

$$
\left\{\begin{array} { l } 
{ \mathfrak { f } _ { j } = \frac { 1 } { 2 } ( e _ { j } - i e _ { m + j } ) , } \\
{ \mathfrak { f } _ { j } ^ { \dagger } = - \frac { 1 } { 2 } ( e _ { j } + i e _ { m + j } ) , }
\end{array} \quad \left\{\begin{array}{l}
\mathfrak{f}_{j}=\frac{1}{2}\left(\grave{e_{2 j-1}}-i \grave{e_{2 j}}\right), \\
\mathfrak{f}_{j}^{\dagger}=-\frac{1}{2}\left(\grave{e_{2 j-1}}+i \grave{e_{2 j}}\right) .
\end{array}\right.\right.
$$

It is easily seen that these Witt basis elements generate the complexification of $\mathcal{C}_{2 m, 2 n}$ and are subject to the following commutation rules

$$
\left\{\begin{array} { l } 
{ \mathfrak { f } _ { j } \mathfrak { f } _ { k } + \mathfrak { f } _ { k } \mathfrak { f } _ { j } = 0 , } \\
{ \mathfrak { f } _ { j } ^ { \dagger } \mathfrak { f } _ { k } ^ { \dagger } + \mathfrak { f } _ { k } ^ { \dagger } \mathfrak { f } _ { j } ^ { \dagger } = 0 , } \\
{ \mathfrak { f } _ { j } \mathfrak { f } _ { k } ^ { \dagger } + \mathfrak { f } _ { k } ^ { \dagger } \mathfrak { f } _ { j } = \delta _ { j , k } , }
\end{array} \quad \left\{\begin{array} { l } 
{ \mathfrak { f } _ { j } \mathfrak { f } _ { k } - \mathfrak { f } _ { k } \mathfrak { f } _ { j } = 0 , } \\
{ \mathfrak { f } _ { j } ^ { \dagger } \mathfrak { f } _ { k } ^ { \dagger } - \mathfrak { f } _ { k } ^ { \dagger } \mathfrak { f } _ { j } ^ { \dagger \dagger } = 0 , } \\
{ \mathfrak { f } _ { j } \mathfrak { f } _ { k } ^ { \dagger } - \mathfrak { f } _ { k } ^ { \dagger } \mathfrak { f } _ { j } = - \frac { i } { 2 } \delta _ { j , k } , }
\end{array} \quad \left\{\begin{array} { l } 
{ \mathfrak { f } _ { j } \mathfrak { f } _ { k } { } ^ { \dagger } + \mathfrak { f } _ { k } \mathfrak { f } _ { j } = 0 , } \\
{ \mathfrak { f } _ { j } \mathfrak { f } _ { k } ^ { \dagger } + \mathfrak { f } _ { k } ^ { \dagger } \mathfrak { f } _ { j } = 0 , }
\end{array} \quad \left\{\begin{array}{l}
\mathfrak{f}_{j}^{\dagger} \mathfrak{f}_{k}+\mathfrak{f}_{k} \mathfrak{f}_{j}^{\dagger}=0 \\
\mathfrak{f}_{j}^{\dagger} \mathfrak{f}_{k}^{\dagger}+\mathfrak{f}_{k}^{\dagger} \mathfrak{f}_{j}^{\dagger}=0
\end{array}\right.\right.\right.\right.
$$

As a consequence of the radial algebra results, the new Hermitian vector variables and the bivector $\mathbf{B}$ satisfy the properties $\left(\mathbf{A H} \mathbf{1}^{*}\right)-\left(\mathbf{A H} \mathbf{3}^{*}\right)$. In particular, $\left\{z, u^{\dagger}\right\}$ is a commuting object in the whole algebra $\mathbb{C} \mathcal{A}_{2 m, 2 n}$ and has the form

$$
\left\{z, u^{\dagger}\right\}=\sum_{j=1}^{m} z_{j} u_{j}^{c}-\frac{i}{2} \sum_{j=1}^{n} \grave{z_{j}} u_{j}^{c} .
$$

The complex conjugation ${ }^{c}$ acts on the complexification of $\mathcal{V}$ as the corresponding restriction of $\cdot{ }^{\dagger}$, whence it is clear that

$$
\left\{z, u^{\dagger}\right\}^{c}=\sum_{j=1}^{m} z_{j}^{c} u_{j}+\frac{i}{2} \sum_{j=1}^{n} z_{j}^{c} \grave{u_{j}}=\sum_{j=1}^{m} u_{j} z_{j}^{c}-\frac{i}{2} \sum_{j=1}^{n} \grave{u}_{j} z_{j}^{c}=\left\{u, z^{\dagger}\right\},
$$

meaning that formula (15) can be used as a generalized inner product.

We also introduce the left and right actions of the Hermitian vector derivatives in this setting using radial algebra notions. These are

$$
\begin{aligned}
& \partial_{z^{\prime}} \cdot=\frac{1}{4}\left(\partial_{\mathbf{x}} \cdot-i \partial_{J(\mathbf{x})} \cdot\right)=\frac{1}{4}\left(\partial_{\underline{x}} \cdot-i \partial_{J(\underline{x})} \cdot\right)-\frac{1}{4}\left(\partial_{\underline{x}} \cdot-i \partial_{J(\underline{x})} \cdot\right)=\partial_{\underline{\underline{z}}} \cdot+\partial_{\underline{z}} \cdot, \\
& \partial_{z^{\dagger}} \cdot=-\frac{1}{4}\left(\partial_{\mathbf{x}} \cdot+i \partial_{J(\mathbf{x})} \cdot\right)=-\frac{1}{4}\left(\partial_{\underline{x}} \cdot+i \partial_{J(\underline{x})} \cdot\right)+\frac{1}{4}\left(\partial_{\underline{x}} \cdot+i \partial_{J(\underline{x})} \cdot\right)=\partial_{\underline{z}^{\dagger}} \cdot+\partial_{\underline{z}^{\dagger}} \cdot, \\
& \cdot \partial_{z}=\frac{1}{4}\left(\cdot \partial_{\mathbf{x}}-i \cdot \partial_{J(\mathbf{x})}\right)=\frac{1}{4}\left(-\cdot \partial_{\underline{x}}+i \cdot \partial_{J(\underline{x})}\right)-\frac{1}{4}\left(\cdot \partial_{\underline{x}}-i \cdot \partial_{J(\underline{x})}\right)=-\cdot \partial_{\underline{z}}+\cdot \partial_{\underline{z}}, \\
& \cdot \partial_{z^{\dagger}}=-\frac{1}{4}\left(\cdot \partial_{\mathbf{x}}+i \cdot \partial_{J(\mathbf{x})}\right)=\frac{1}{4}\left(\cdot \partial_{\underline{x}}+i \cdot \partial_{J(\underline{x})}\right)+\frac{1}{4}\left(\cdot \partial_{\underline{x}}+i \cdot \partial_{J(\underline{x})}\right)=-\cdot \partial_{\underline{z}^{\dagger}}+\cdot \partial_{\underline{z}^{\dagger}} \cdot
\end{aligned}
$$


Meanwhile, we have introduced the bosonic and fermionic Hermitian Dirac operators

$$
\partial_{\underline{z}}=-\frac{1}{4}\left(\partial_{\underline{x}}-i \partial_{J(\underline{x})}\right)=\sum_{j=1}^{m} \mathfrak{f}_{j}^{\dagger} \partial_{z_{j}}, \quad \partial_{\underline{z}}=\frac{1}{4}\left(\partial_{\underline{x}}-i \partial_{J(\underline{x})}\right)=2 i \sum_{j=1}^{n} \mathfrak{f}_{j}^{\dagger} \partial_{\grave{z}_{j}},
$$

and their Hermitian conjugates

$$
\partial_{\underline{z}^{\dagger}}=\frac{1}{4}\left(\partial_{\underline{x}}+i \partial_{J(\underline{x})}\right)=\sum_{j=1}^{m} \mathfrak{f}_{j} \partial_{z_{j}^{c}}, \quad \partial_{\underline{z}^{\dagger}}=-\frac{1}{4}\left(\partial_{\underline{x}}+i \partial_{J(\underline{x})}\right)=-2 i \sum_{j=1}^{n} \mathfrak{f}_{j} \partial_{z_{j}^{c}},
$$

where

$$
\partial_{z_{j}}=\frac{1}{2}\left(\partial_{x_{j}}-i \partial_{x_{m+j}}\right), \quad \partial_{z_{j}^{c}}=\frac{1}{2}\left(\partial_{x_{j}}+i \partial_{x_{m+j}}\right), \quad \partial_{\bar{z}_{j}}=\frac{1}{2}\left(\partial_{\grave{x_{2 j-1}}}-i \partial_{\grave{x_{2 j}}}\right), \quad \partial_{\check{z}_{j}^{c}}=\frac{1}{2}\left(\partial_{\grave{x_{2 j-1}}}+i \partial_{\grave{x_{2 j}}}\right),
$$

are the classical Cauchy-Riemann operators and their conjugates with respect to the variables $z_{j}$ and $\grave{z}_{j}$.

As it was shown in [8], the operators $\partial_{z}$ and $\partial_{z^{\dagger}}$ satisfy the relations $\left(\mathbf{D H} \mathbf{1}^{*}\right)-\left(\mathbf{D H} \mathbf{4}^{*}\right)$ given in Section 2. These relations can be also checked using the above explicit expressions, e.g.:

$$
\partial_{z}[z]=\partial_{\underline{z}[}[\underline{z}]+\partial_{\underline{z}}[\underline{z}]=2 i \sum_{j=1}^{n} \mathfrak{f}_{j}^{\dagger} \mathfrak{f}_{j}+\sum_{j=1}^{m} \mathfrak{f}_{j}^{\dagger} \mathfrak{f}_{j}=\frac{1}{2}[(m-n)+i \mathbf{B}] .
$$

We also obtain explicit formulas for the complex directional derivatives in superspace following the radial algebra approach. In fact, for every pair of Hermitian vector variables $z=\frac{1}{2}(\mathbf{x}+i J(\mathbf{x}))$ and $u=$ $\frac{1}{2}(\mathbf{y}+i J(\mathbf{y}))$ we have

$$
\begin{aligned}
& D_{u, z}=\frac{1}{2}\left(D_{\mathbf{y}, \mathbf{x}}+i D_{J(\mathbf{y}), \mathbf{x}}\right)=\frac{1}{2}\left[\left(D_{\underline{y}, \underline{x}}+i D_{J(\underline{y}), \underline{x}}\right)+\left(D_{\underline{y}, \underline{x}}+i D_{J(\underline{y}), \underline{x}}\right)\right]=D_{\underline{u}, \underline{z}}+D_{\underline{u}, \underline{\underline{z}}}, \\
& D_{u, z}^{\dagger}=\frac{1}{2}\left(D_{\mathbf{y}, \mathbf{x}}-i D_{J(\mathbf{y}), \mathbf{x}}\right)=\frac{1}{2}\left[\left(D_{\underline{y}, \underline{x}}-i D_{J(\underline{y}), \underline{x}}\right)+\left(D_{\underline{y}, \underline{x}}-i D_{J(\underline{y}), \underline{x}}\right)\right]=D_{\underline{u}, \underline{z}}^{\dagger}+D_{\underline{\underline{u}}, \underline{\underline{z}}}^{\dagger} .
\end{aligned}
$$

where the bosonic and fermionic directional derivatives and their Hermitian conjugates are given by

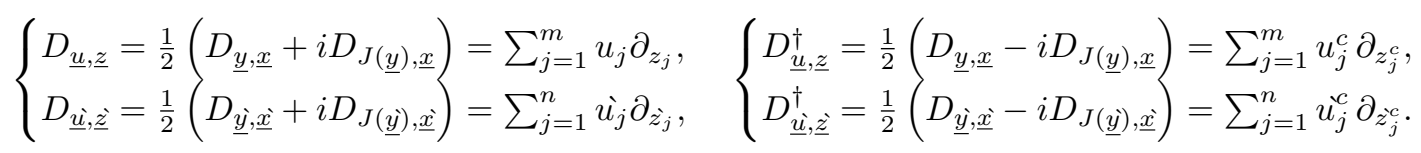

As a consequence of the results obtained at the radial algebra level we have

$$
\left\{\partial_{z}, u\right\}=D_{u, z}+\frac{1}{2} \delta_{z, u}((m-n)+i \mathbf{B}), \quad\left\{\partial_{z^{\dagger}}, u^{\dagger}\right\}=D_{u, z}^{\dagger}+\frac{1}{2} \delta_{z, u}((m-n)-i \mathbf{B}),
$$

and it can be easily checked that the above relations remain valid in $\mathbb{C} \mathcal{A}_{2 m, 2 n}$.

In the case, $z=u$ we obtain the Hermitian Euler operators

$$
\mathbb{E}_{z}=D_{z, z}=\sum_{j=1}^{m} z_{j} \partial_{z_{j}}+\sum_{j=1}^{n} \grave{z_{j}} \partial_{\check{z}_{j}} \quad \text { and } \quad \mathbb{E}_{z^{\dagger}}=D_{z, z}^{\dagger}=\sum_{j=1}^{m} z_{j}^{c} \partial_{z_{j}}^{c}+\sum_{j=1}^{n} z_{j}^{c} \partial_{z_{j}^{c}}
$$

which split the Euler operator $\mathbb{E}_{x}$ as $\mathbb{E}_{x}=\mathbb{E}_{z}+\mathbb{E}_{z^{\dagger}}$.

It was shown in [8] that $\mathbb{E}_{z}$ and $\mathbb{E}_{z}^{\dagger}$ measure the degree of homogeneity of the vector variables $z$ and $z^{\dagger}$ respectively on every element of the Hermitian radial algebra. As expected, also this property generalizes to $\mathbb{C} \mathcal{A}_{2 m, 2 n}$.

To this end, we refine the notion of a $k$-homogeneous polynomial to a (bi-)homogeneous polynomial of degree $(p, q)$ with $p+q=k$. A polynomial $R_{p, q}\left(z, z^{\dagger}\right)=R_{p, q}\left(z_{1}, \ldots, z_{m}, z_{1}^{c}, \ldots, z_{m}^{c}, \grave{z}_{1}, \ldots, \grave{z}_{n}, z_{1}^{c}, \ldots, z_{n}^{c}\right)$ is said to be homogeneous of degree $(p, q) \in \mathbb{N}^{2}$ if, for all $\lambda \in \mathbb{C} \backslash\{0\}$ it holds that

$$
R_{p, q}\left(\lambda z, \lambda^{c} z^{\dagger}\right)=\lambda^{p}\left(\lambda^{c}\right)^{q} R_{p, q}\left(z, z^{\dagger}\right) .
$$

The space of all homogeneus polynomials of degree $(p, q)$ in $\mathcal{P}$ is denoted by $\mathcal{P}_{p, q}$. A basis for $\mathcal{P}_{p, q}$ consists of the elements $z_{1}^{\alpha_{1}} \cdots z_{m}^{\alpha_{m}} z_{1}^{\beta_{1}} \cdots z_{n}^{\beta_{n}}\left(z_{1}^{c}\right)^{\gamma_{1}} \cdots\left(z_{m}^{c}\right)^{\gamma_{m}}\left(z_{1}^{c}\right)^{\delta_{1}} \cdots\left(z_{n}^{c}\right)^{\delta_{n}}$ where $\alpha_{j}, \gamma_{j} \in \mathbb{N}, \beta_{j}, \delta_{j} \in\{0,1\}$ 
and with $\sum_{j=1}^{m} \alpha_{j}+\sum_{j=1}^{n} \beta_{j}=p, \sum_{j=1}^{m} \gamma_{j}+\sum_{j=1}^{n} \delta_{j}=q$. It easily follows that $\mathcal{P}_{p, q}$ is a finite dimensional complex vector space with dimension

$$
\operatorname{dim} \mathcal{P}_{p, q}=\left[\sum_{j=0}^{\min (n, p)}\left(\begin{array}{l}
n \\
j
\end{array}\right)\left(\begin{array}{c}
p-j+m-1 \\
m-1
\end{array}\right)\right]\left[\sum_{j=0}^{\min (n, q)}\left(\begin{array}{l}
n \\
j
\end{array}\right)\left(\begin{array}{c}
q-j+m-1 \\
m-1
\end{array}\right)\right] .
$$

Since each complex polynomial in $\left(x_{1}, \ldots, x_{2 m}, \grave{x_{1}}, \ldots, \grave{x_{2 n}}\right)$ may be written also as a polynomial in the variables $\left(z_{1}, \ldots, z_{m}, z_{1}^{c}, \ldots, z_{m}^{c}, \grave{z_{1}}, \ldots, \grave{z}_{n}, \grave{z}_{1}^{c}, \ldots, z_{n}^{c}\right)$, we can easily check the relation

$$
\mathcal{P}_{k}=\bigoplus_{j=0}^{k} \mathcal{P}_{j, k-j}
$$

In the previous section, it was mentioned that $\mathcal{P}_{k}$ is the eigenspace of $\mathbb{E}_{x}$ corresponding to the eigenvalue $k$. A similar property can be proved in the Hermitian context.

Lemma 3. If $R_{p, q}\left(z, z^{\dagger}\right)$ is a homogeneous polynomial of degree $(p, q)$ then

$$
\mathbb{E}_{z}\left[R_{p, q}\right]=p R_{p, q}, \quad \text { and } \quad \mathbb{E}_{z^{\dagger}}\left[R_{p, q}\right]=q R_{p, q}
$$

Proof.

Applying the chain rule (see [6]) and differentiating with respect to the complex variable $\lambda$, we have on the one hand

$$
\partial_{\lambda} R_{p, q}\left(\lambda z, \lambda^{c} z^{\dagger}\right)=\sum_{j=1}^{m} z_{j} \partial_{z_{j}} R_{p, q}\left(\lambda z, \lambda^{c} z^{\dagger}\right)+\sum_{j=1}^{n} \grave{z_{j}} \partial_{z_{j}} R_{p, q}\left(\lambda z, \lambda^{c} z^{\dagger}\right)=\mathbb{E}_{z} R_{p, q}\left(\lambda z, \lambda^{c} z^{\dagger}\right),
$$

and on the other hand,

$$
\partial_{\lambda} R_{p, q}\left(\lambda z, \lambda^{c} z^{\dagger}\right)=\partial_{\lambda}\left[\lambda^{p}\left(\lambda^{c}\right)^{q} R_{p, q}\left(z, z^{\dagger}\right)\right]=p \lambda^{p-1}\left(\lambda^{c}\right)^{q} R_{p, q}\left(z, z^{\dagger}\right),
$$

whence,

$$
\mathbb{E}_{z} R_{p, q}\left(\lambda z, \lambda^{c} z^{\dagger}\right)=p \lambda^{p-1}\left(\lambda^{c}\right)^{q} R_{p, q}\left(z, z^{\dagger}\right) .
$$

In particular, for $\lambda=1$ we have $\mathbb{E}_{z}\left[R_{p, q}\right]=p R_{p, q}$. The proof of the other relation is similar.

\section{Conclusions and Further Research}

We have carefully introduced the defining objects for Hermitian Clifford analysis in superspace through the rules determined by the Hermitian radial algebra. These rules provide a straightforward way of defining a suitable complex structure in this setting, giving rise to the introduction of all basic elements in the Hermitian Clifford calculus. This complex structure can be seen either as a special automorphism on $\mathcal{A}_{2 m, 2 n}$ or as the action of the special bivector $\mathbf{B}$ through its commutator with vector variables. This action of $\mathbf{B}$ allows to interprete the complex structure as a special element of the set of superrotations. In forthcoming work we will further develop this theory. This will include a deep study of the group realization of rotations in superspace and the invariance of the super Dirac operators under the action of these groups $([9,10])$. Also a Bochner-Martinelli formula in this setting will be established.

\section{Acknowledgements}

Alí Guzmán Adán is supported by a BOF-doctoral grant from Ghent University with grant number $01 \mathrm{D} 06014$. 


\section{References}

[1] F. Brackx, H. De Schepper, and V. Souček. Fischer decompositions in euclidean and hermitean clifford analysis. Archivum Mathematicum, 46(5):301-321, 2010.

[2] Fred Brackx, Jarolím Bureš, Hennie De Schepper, David Eelbode, Frank Sommen, and Vladímir Souček. Fundaments of hermitean clifford analysis part i: Complex structure. Complex Analysis and Operator Theory, 1(3):341-365, 2007.

[3] Fred Brackx, Hennie De Schepper, and Frank Sommen. The Hermitian Clifford analysis toolbox. Adv. Appl. Clifford Algebr., 18(3-4):451-487, 2008.

[4] F. Colombo, I. Sabadini, F. Sommen, and D. C. Struppa. Analysis of Dirac systems and computational algebra, volume 39 of Progress in Mathematical Physics. Birkhäuser Boston, Inc., Boston, MA, 2004.

[5] H. De Bie and F. Sommen. A clifford analysis approach to superspace. Annals of Physics, 322(12):2978-2993, 2007.

[6] H. De Bie and F. Sommen. Correct rules for clifford calculus on superspace. Advances in Applied Clifford Algebras, 17(3):357-382, 2007.

[7] H. De Bie, F. Sommen, and M. Wutzig. Reproducing kernels for polynomial null-solutions of dirac operators. Constructive Approximation, 44(3):339-383, 2016.

[8] H. De Schepper, A. Adán Guzmán, and F. Sommen. The Radial Algebra as an Abstract Framework for Orthogonal and Hermitian Clifford Analysis. Complex Anal. Oper. Theory, 11(5):1139-1172, 2017.

[9] H. De Schepper, A. Guzmán Adán, and F. Sommen. The spin group in superspace. Submitted for publication, 2017.

[10] H. De Schepper, A. Guzmán Adán, and F. Sommen. Spin actions in Euclidean and Hermitian Clifford analysis in superspace. J. Math. Anal. Appl., 457(1):23-50, 2018.

[11] R. Delanghe, F. Sommen, and V. Sou cek. Clifford algebra and spinor-valued functions, volume 53 of Mathematics and its Applications. Kluwer Academic Publishers Group, Dordrecht, 1992. A function theory for the Dirac operator, Related REDUCE software by F. Brackx and D. Constales, With 1 IBM-PC floppy disk (3.5 inch).

[12] D. Hestenes and G. Sobczyk. Clifford algebra to geometric calculus. Fundamental Theories of Physics. D. Reidel Publishing Co., Dordrecht, 1984. A unified language for mathematics and physics.

[13] I. Sabadini and F. Sommen. Hermitian clifford analysis and resolutions. Mathematical Methods in the Applied Sciences, 25(16-18):1395-1413, 2002.

[14] I. Sabadini, F. Sommen, and D. C Struppa. The dirac complex on abstract vector variables: megaforms. Experimental mathematics, 12(3):351-364, 2003.

[15] F. Sommen. An algebra of abstract vector variables. In Portugaliae Mathematica 54 (3) (1997), pages 287-310, 1997.

[16] F. Sommen. The problem of defining abstract bivectors. Results in mathematics, 31(1-2):148-160, 1997.

[17] F. Sommen. Clifford analysis on super-space. Advances in Applied Clifford Algebras, 11(1):291-304, 2001.

[18] F. Sommen. Clifford analysis on super-space. ii. Progress in analysis, 1:383-405, 2003. 
[19] Frank Sommen. An extension of Clifford analysis towards super-symmetry. In Clifford algebras and their applications in mathematical physics, Vol. 2 (Ixtapa, 1999), volume 19 of Progr. Phys., pages 199-224. Birkhäuser Boston, Boston, MA, 2000. 